\title{
Asymptotic Stability of Rarefaction Wave for the Navier-Stokes Equations for a Compressible Fluid in the Half Space
}

\author{
Shuichi Kawashima \& Peicheng Zhu*
}

Communicated by Y. BRENIER

\begin{abstract}
This paper is concerned with the asymptotic stability towards a rarefaction wave of the solution to an outflow problem for the Navier-Stokes equations in a compressible fluid in the Eulerian coordinate in the half space. This is the second one of our series of papers on this subject. In this paper, firstly we classify completely the time-asymptotic states, according to some parameters, that is the spatial-asymptotic states and boundary conditions, for this initial boundary value problem, and some pictures for the classification of time-asymptotic states are drawn in the state space. In order to prove the stability of the rarefaction wave, we use the solution to Burgers' equation to construct a suitably smooth approximation of the rarefaction wave and establish some time-decay estimates in $L^{p}$-norm for the smoothed rarefaction wave. We then employ the $L^{2}$-energy method to prove that the rarefaction wave is nonlinearly stable under a small perturbation, as time goes to infinity.
\end{abstract}

\section{Introduction}

In this article, we shall investigate the large-time behavior of the solution to an outflow problem for the one-dimensional isentropic Navier-Stokes equations for a compressible fluid in the Eulerian coordinate in the half space. It will be proved that the solution exists uniquely and converges to a rarefaction wave as time goes to infinity under some suitable assumptions. This is one of the series of papers by the authors on this topic. For the so-called outflow (inflow) problems, we refer to the paper by Matsumura [21] for more details. We are now going to formulate our problem. The one-dimensional and isentropic motion of compressible viscous

\footnotetext{
^ P. Zhu was supported by JSPS postdoctoral fellowship under P99217.
} 
gas, which is confined in the half space $\mathbb{R}^{+}:=\{x \in \mathbb{R} \mid x>0\}$, can be described by the following system:

$$
\begin{aligned}
\rho_{t}+(\rho u)_{x} & =0, \\
(\rho u)_{t}+\left(\rho u^{2}+p(\rho)\right)_{x} & =\left(\mu u_{x}\right)_{x},
\end{aligned}
$$

which must be satisfied in the domain $\{(t, x) \mid t>0, x>0\}$. Here $\rho(>0), u$ and $p$ are the mass density, the velocity and the pressure of the gas, respectively. And $p$ is assumed to be a function of $\rho$ defined by

$$
p=p(\rho)=K \rho^{\gamma} \text {. }
$$

All the above coefficients $\mu(>0), K(>0)$ and $\gamma(>1)$ are assumed to be constants, and $\mu$ is the viscosity coefficient. We do not consider here the linear case, that is $\gamma=1$. The gas corresponding to this case is ideal. We study the initial-boundary value problem to the system (1.1) and (1.2) with the following initial data

$$
(\rho, u)(0, x)=\left(\rho_{0}, u_{0}\right)(x), \text { for all } x>0, \text { and } \inf _{x>0} \rho_{0}(x)>0,
$$

the boundary condition at the infinity $x=+\infty$

$$
\lim _{x \rightarrow \infty}(\rho, u)(t, x)=\left(\rho_{+}, u_{+}\right), \quad \text { for any } t \geqq 0,
$$

and also the boundary conditions at $x=0$

$$
u(t, 0)=u_{b}, \quad \text { for any } t \geqq 0 .
$$

Here, $\rho_{+}, u_{+}, u_{b}$ are given constants satisfying

$$
\rho_{+}>0, \quad u_{b}<0
$$

and $\rho_{0}(x), u_{0}(x)$ are given functions.

We are interested in the so-called outflow problem. For this case the boundary data is taken as $u_{b}<0$. This means physically that the outflow exists constantly thorough the wall. We note that for the case that $u_{b}>0$, the situation is different and the corresponding problem is called an inflow problem. In that case, for the well-posedness, one must impose one more boundary condition at $x=0$, namely we must consider a set of boundary conditions of the form

$$
\rho(t, 0)=\rho_{b}, \quad u(t, 0)=u_{b}, \quad t \geqq 0 .
$$

with $\rho_{b}>0$ and $u_{b}>0$. Such an inflow problem is also interesting and has been studied partly by Matsumura AND NishiHara [26], and so on.

We consider the asymptotic stability towards non-linear waves of the solution to the outflow problem under the space-asymptotic condition (1.4). Moreover, we assume for simplicity that 


$$
\rho>0 \text { and } u_{+} \neq u_{b}
$$

where the first condition in (1.7) means that no vacuum state is allowed, and the second one is assumed to avoid some technical difficulties. It can be expected that as $t \rightarrow \infty$, the solution $(\rho, u)$ to the above problem (1.1)-(1.5) is asymptotically described by one of the following waves, such as a viscous shock wave, a stationary wave, a rarefaction wave or the superposition of a stationary wave and a rarefaction wave, which can be determined by the space-asymptotic conditions (1.4) and the boundary data $u_{b}$. The stability of a stationary wave has been investigated by KawASHIMA et al. [10]. The study of the stability of a viscous shock wave or the superposition of a rarefaction wave and a stationary wave will be carried out in other papers by the authors. In this paper, we are interested particularly in the case that the corresponding time-asymptotic state is rarefaction wave, that is sub-case 2 of Case (C) and sub-case 1 of Case (D), see Section 1.1 below.

\subsection{Classification of the time-asymptotic states}

In this subsection, we shall classify the time-asymptotic states to the initial boundary value problem (1.1)-(1.5). The system (1.1) and (1.2) with $\mu=0$ has two distinct characteristic fields, since we assume that $\rho>0$, which correspond the following two eigenvalues:

$$
\lambda_{1}(\rho, u)=u-C(\rho), \quad \lambda_{2}(\rho, u)=u+C(\rho) .
$$

Here $C(\rho)$ is the sound speed defined by

$$
C(\rho)=\sqrt{\gamma K \rho^{\gamma-1}}
$$

We introduce a new function $M=\frac{|u|}{C(\rho)}$, which is called the Mach number. We need especially the quantity at infinity

$$
M_{+}=\frac{\left|u_{+}\right|}{C\left(\rho_{+}\right)} \text {. }
$$

For our outflow problem, the second characteristic field that corresponds to the second eigenvalues $\lambda_{2}(\rho, u)$ is important. In fact, it is convenient to classify the time-asymptotic states, according to the sign of $\lambda_{2}(\rho, u)$ at $\left(\rho_{+}, u_{+}\right)$. We classify it into four cases, and each case is classified furthermore into several sub-cases:
(A) $u_{+}<-C\left(\rho_{+}\right)$, ( that is $\lambda_{2}\left(\rho_{+}, u_{+}\right)<0$ and $\left.M_{+}>1\right)$,
(B) $u_{+}=-C\left(\rho_{+}\right)$, ( that is $\lambda_{2}\left(\rho_{+}, u_{+}\right)=0$ and $\left.M_{+}=1\right)$,
(C) $-C\left(\rho_{+}\right)<u_{+}<0$, ( that is $\lambda_{2}\left(\rho_{+}, u_{+}\right)>0$ with $u_{+}<0$ and $\left.M_{+}<1\right)$,
(D) $u_{+}>0$. 
It is easy to see that the values of $u_{+}$in the first three cases are taken negative; however $u_{+}$is positive in the fourth case. Clearly, Case A) is the case that $\left(\rho_{+}, u_{+}\right)$ is in the supersonic region, where the Mach number $M_{+}>1$, in the state space with $u_{+}<0$, while for Cases (B) or (C) the state $\left(\rho_{+}, u_{+}\right)$is in the transonic $\left(M_{+}=1\right)$ or subsonic $\left(M_{+}<1\right)$ region, respectively. In what follows we present a complete classification of the time-asymptotic states of the solution $(\rho, u)$ by regarding the boundary data $u_{b}, u_{+}, v_{+}$as parameters. From now on, we define

$$
v=\frac{1}{\rho}, \quad v_{+}=\frac{1}{\rho_{+}}, \ldots, \quad \text { and so on, }
$$

where $v$ is the specific volume. Correspondingly, we introduce a new function

$$
\hat{C}(v):=C\left(\frac{1}{v}\right) .
$$

We now draw the pictures in the state space which help us to understand this classification of the non-linear waves.

Case (A): For the supersonic case, $u_{+}<-\hat{C}\left(v_{+}\right)$, we have that

There are three sub-cases for this case:

Case 1. $u_{b}<u_{*}$ : There exists a unique stationary solution $(\tilde{\rho}, \tilde{u})(x)$ such that

$$
\tilde{u}=\frac{u_{+}}{v_{+}} \tilde{v}
$$

with $\tilde{u}(0)=u_{b}$ and $(\tilde{\rho}, \tilde{u})(x) \rightarrow\left(\rho_{+}, u_{+}\right)$as $x \rightarrow \infty$. $\tilde{\rho}(0)=1 / v_{b}$ and $v_{b}$ is determined by the formula $u_{b}=\frac{u_{+}}{v_{+}} v_{b}$. Moreover, we have either (i) If $u_{b}<u_{+}$, then $\tilde{u}_{x}>0$, that is $\tilde{u}$ is monotonously increasing in $x$, or (ii) If $u_{+}<u_{b}<u_{*}$, then $\tilde{u}_{x}<0$, that is $\tilde{u}$ is monotonously decreasing in $x$.

Case 2. $u_{b}=u_{*}$ : There exists a 2 -shock profile $(\tilde{\rho}, \tilde{u})(x)$ with speed $s=0$ which connects the two states $\left(v_{*}, u_{*}\right)$ and $\left(v_{+}, u_{+}\right)$, and

$$
\begin{aligned}
& (\tilde{\rho}, \tilde{u})(x) \rightarrow\left(\rho_{+}, u_{+}\right), \quad \text { as } x \rightarrow \infty \\
& (\tilde{\rho}, \tilde{u})(x) \rightarrow\left(\rho_{*}, u_{*}\right), \quad \text { as } x \rightarrow-\infty
\end{aligned}
$$

Case 3. $u_{*}<u_{b}<0$ : We choose $\left(v_{-}, u_{b}\right) \in S_{2}\left(S_{2}\right.$ is the 2-shock curve, defined by $S_{2}: u-u_{b}=-\sqrt{\left(v-v_{-}\right)\left(p\left(v_{-}\right)-p(v)\right)}$ for $\left.v_{-}<v\right)$, then there exists a 2 -shock profile $(\tilde{\rho}, \tilde{u})(x-s t)$ with positive speed $s>0$, which connects $\left(v_{-}, u_{b}\right)$ and $\left(v_{+}, u_{+}\right)$. Hereafter, we denote $\xi:=x-s t$. We find the following equation:

$$
\begin{aligned}
& (\tilde{\rho}, \tilde{u})(\xi) \rightarrow\left(\rho_{+}, u_{+}\right), \quad \text { as } \xi \rightarrow \infty \\
& (\tilde{\rho}, \tilde{u})(\xi) \rightarrow\left(\rho_{-}, u_{b}\right), \quad \text { as } \xi \rightarrow-\infty
\end{aligned}
$$




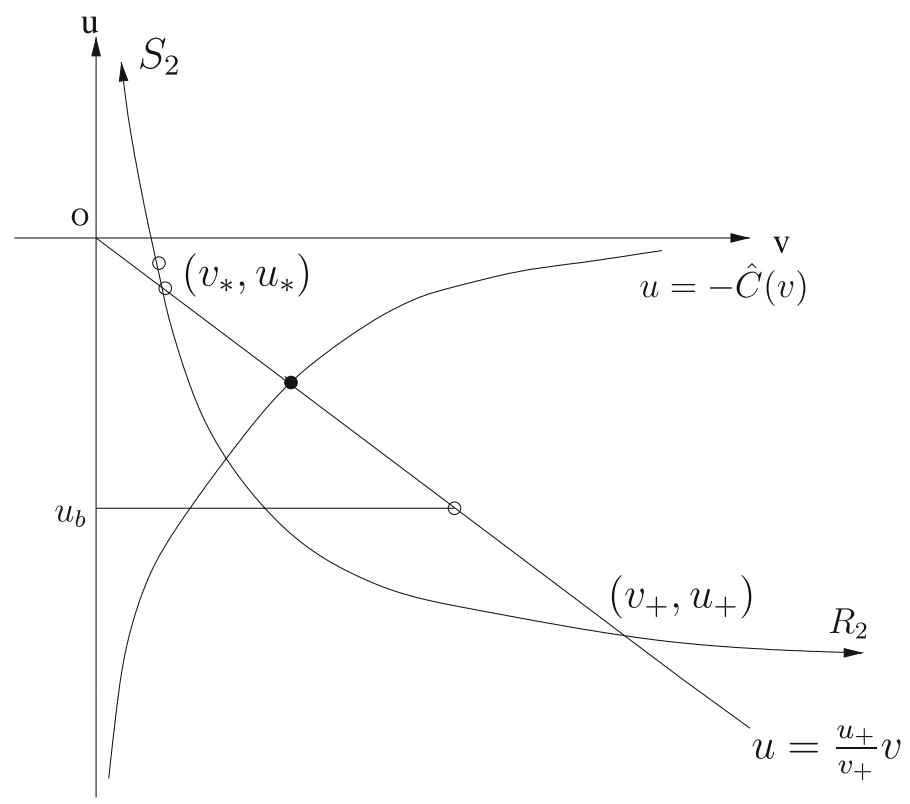

Fig. A.

Case (B): The transonic case, that is $u_{+}=-\hat{C}\left(v_{+}\right)$. For this case, the values $\left(v_{*}, u_{*}\right),\left(v_{+}, u_{+}\right)$, which appeared in Case A), satisfy that they now coincide, that is $v_{*}=v_{+}, u_{*}=u_{+}$. Therefore, for this case, we can divide it into two sub-cases:

Case 1. $u_{b}<u_{+}$: There exists a unique stationary solution $(\tilde{\rho}, \tilde{u})(x)$ satisfying

$$
\tilde{u}=\frac{u_{+}}{v_{+}} \tilde{v}, \quad \tilde{u}_{x}>0
$$

with $\tilde{u}(0)=u_{b}$ and $(\tilde{\rho}, \tilde{u})(x) \rightarrow\left(\rho_{+}, u_{+}\right)$as $x \rightarrow \infty$. $\tilde{\rho}(0)=1 / v_{b}$, where $v_{b}$ is determined by the relation

$$
u_{b}=\frac{u_{+}}{v_{+}} v_{b}
$$

Case 2. $u_{+}<u_{b}<0$ : We choose $\left(v_{-}, u_{b}\right) \in S_{2}$, then there exists 2-shock profile $(\tilde{\rho}, \tilde{u})(\xi)$ with $s>0$, which connects $\left(v_{-}, u_{b}\right)$ and $\left(v_{+}, u_{+}\right)$, and satisfies

$$
\begin{aligned}
& (\tilde{\rho}, \tilde{u})(\xi) \rightarrow\left(\rho_{+}, u_{+}\right), \quad \text { as } \xi \rightarrow \infty \\
& (\tilde{\rho}, \tilde{u})(\xi) \rightarrow\left(\rho_{-}, u_{b}\right), \quad \text { as } \xi \rightarrow-\infty
\end{aligned}
$$


The picture for this case now becomes

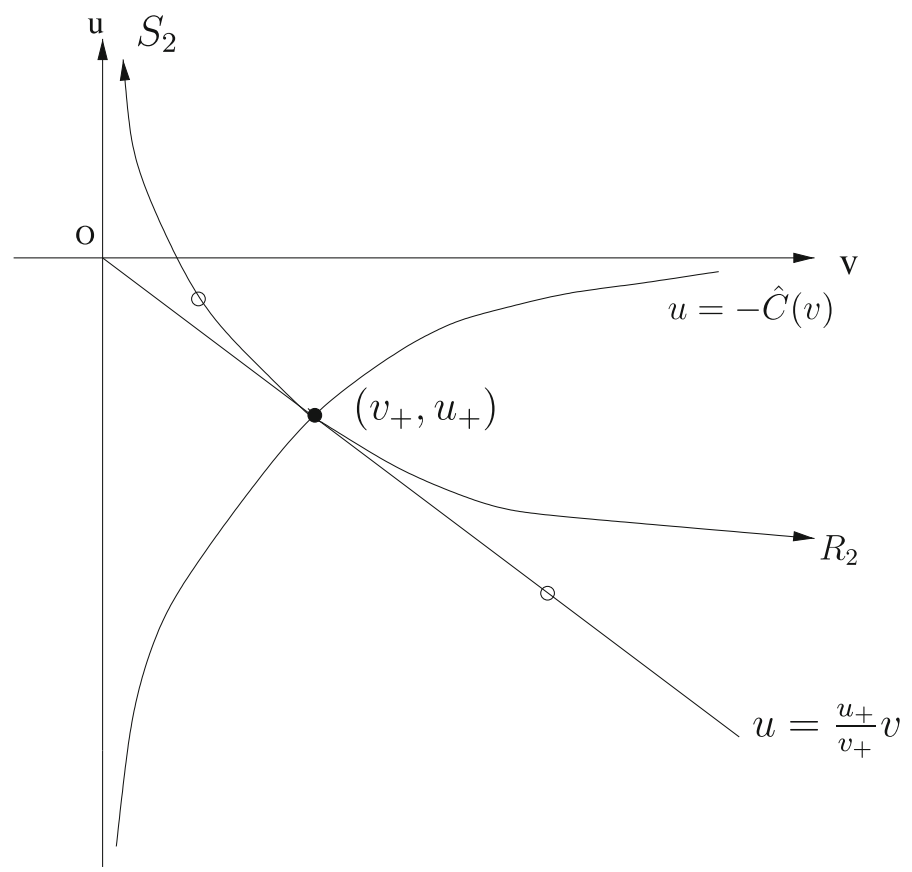

Fig. B.

Case (C): The subsonic case. There holds $-\hat{C}\left(v_{+}\right)<u_{+}<0$.

This case can be divided into three sub-cases:

Case 1. $u_{+}<u_{b}<0$ : We choose $\left(v_{-}, u_{b}\right) \in S_{2}$, then there exists a 2-shock profile $(\tilde{\rho}, \tilde{u})(\xi)$ with $s>0$, which connects $\left(v_{-}, u_{b}\right)$ and $\left(v_{+}, u_{+}\right)$. Moreover there holds

$$
\begin{aligned}
& (\tilde{\rho}, \tilde{u})(\xi) \rightarrow\left(\rho_{+}, u_{+}\right), \quad \text { as } \xi \rightarrow \infty ; \\
& (\tilde{\rho}, \tilde{u})(\xi) \rightarrow\left(\rho_{-}, u_{b}\right), \quad \text { as } \xi \rightarrow-\infty
\end{aligned}
$$

Case 2. $u_{*} \leqq u_{b}<u_{+}$: We choose $\left(v_{-}, u_{b}\right) \in R_{2}$ ( $R_{2}$ is the 2-rarefaction curve, defined by $R_{2}: u-u_{b}=-\int_{v_{-}}^{v} \sqrt{-p^{\prime}(y)} d y$ for $\left.v_{-}>v\right)$, then there exists a 2-rarefaction wave $\left(\rho^{R}, u^{R}\right)(x / t)$ (with $\left.\lambda_{2} \geqq 0\right)$, which connects $\left(v_{-}, u_{b}\right)$ and $\left(v_{+}, u_{+}\right)$, to the corresponding Riemann problem.

Case 3. $u_{b}<u_{*}$ : We choose $\left(v_{b}, u_{b}\right) \in \mathcal{L}$, here $\mathcal{L}$ is a line defined by $\mathcal{L}:=$ $\left\{(v, u) ; u=\frac{u_{*}}{v_{*}} v, v>0\right\}$. Then the time-asymptotic state is the superposition of the rarefaction wave and the stationary solution: 


$$
\left(\rho^{\text {Asym }}, u^{\text {Asym }}\right)(x, t):=(\tilde{\rho}, \tilde{u})(x)+\left(\rho^{R}, u^{R}\right)\left(\frac{x}{t}\right)-\left(\rho_{*}, u_{*}\right),
$$

where $(\tilde{\rho}, \tilde{u})(x)$ is the stationary solution connecting $\left(v_{-}, u_{b}\right)$ and $\left(v_{*}, u_{*}\right)$, while $\left(\rho^{R}, u^{R}\right)\left(\frac{x}{t}\right)$ is the 2-rarefaction wave which connects $\left(v_{*}, u_{*}\right)$ and $\left(v_{+}, u_{+}\right)$.

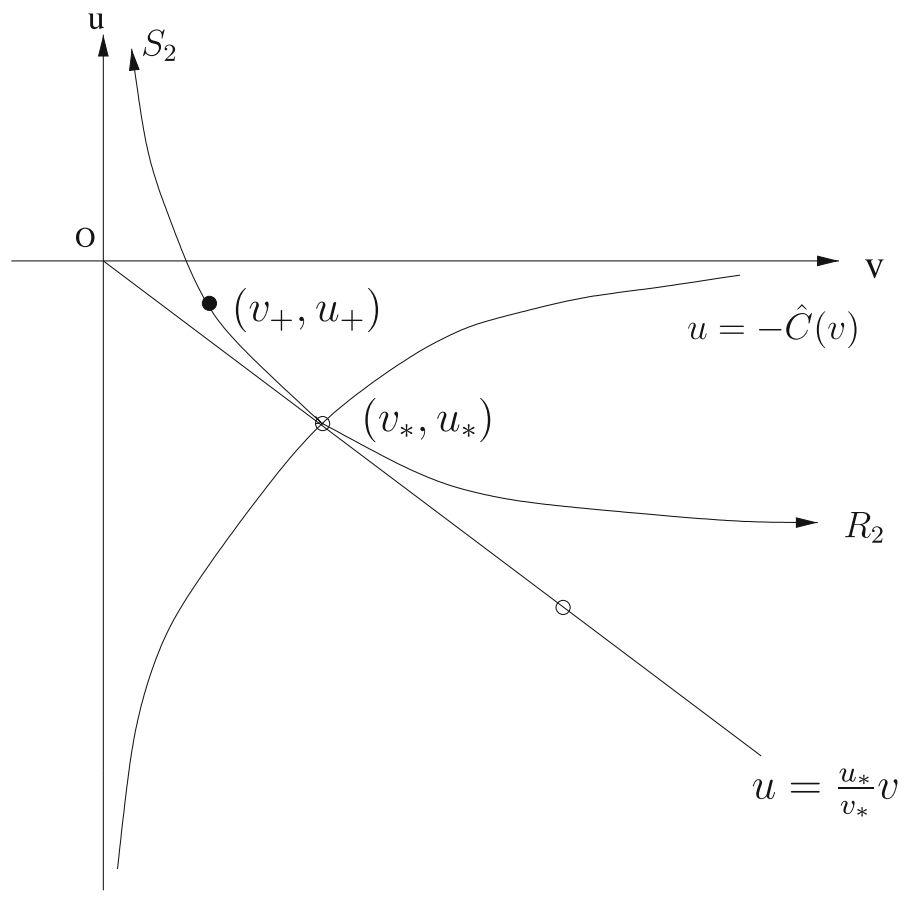

Fig. C.

Case (D): For $u_{+}>0$, we have

This case also can be divided into three sub-cases:

Case 1. $u_{*} \leqq u_{b}<0$ : Taking $\left(v_{-}, u_{b}\right) \in R_{2}$, we then assert that there exists 2-rarefaction wave to the corresponding Riemann problem. The same as sub-Case 2 of Case (C).

Case 2. $u_{b}<u_{*}$ : We choose $\left(v_{b}, u_{b}\right) \in \mathcal{L}\left(\right.$ Here $\left.\mathcal{L}:=\left\{(v, u) ; u=\frac{u_{*}}{v_{*}} v, v>0\right\}\right)$, then the time-asymptotic state is

$$
\left(\rho^{\text {Asym }}, u^{\text {Asym }}\right)(x, t):=(\tilde{\rho}, \tilde{u})(x)+\left(\rho^{R}, u^{R}\right)\left(\frac{x}{t}\right)-\left(\rho_{*}, u_{*}\right) .
$$

The same as sub-Case 3 of Case (C). 


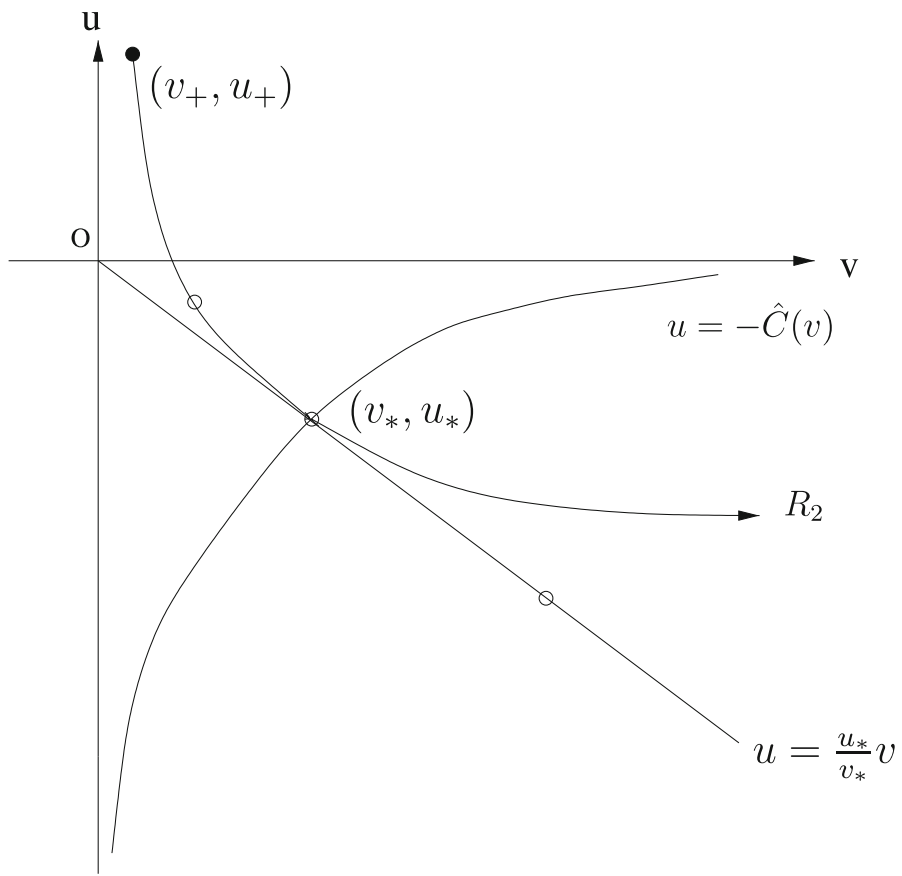

Fig. D.

From the above pictures we can easily see various types of time-asymptotic states corresponding to the different values of parameters. The classification for the inflow problem we refer to Matsumura [21]. We should mention here that the corresponding classification of time-asymptotic states for the non-isentropic problem (which is a $3 \times 3$-system) is much more complicated and remains open up to now.

We now turn to recall some references related to our paper. In [5] IL'IN and OLEINIK pioneered the study of stability of non-linear waves to the Cauchy problems for scalar conservation laws. Since then there have been many mathematicians who have continued this study, see, for example FreISTÜHLER and SERre [1], GoOdman [3], Jones et al. [6], Matsumura and Nishihara [27], Mei [29]. For this kind of study on Cauchy problems for the $p$-system and more general systems, we refer the readers to Kawashima and Matsumura [7,8], Liu [15], LiU and Xin [20], Matsumura and Nishihara [22-24], etc.

As for the initial boundary value problems, we shall mention LiU et al. [17], LiU and NishiHara [18], LiU and YU [19] for the scalar case. However, the following papers are concerned with the study on the system of equations: KAWASHIMA and Nikkuni [9], Kawashima and Tanaka [12], Matsumura and Mei [28], Matsumura and Nishimara [25], etc. Especially, for the isentropic compressible Navier-Stokes equations, MATSUMURA and NishiHARA [26] study partly the inflow problem, while KaWASHima et al. [10], and KawAShima and ZHU [30] investigate the outflow problem. For the non-isentropic compressible Navier-Stokes system, 
under the supervision of the first author, ZHU [30,31] prove the existence and stability of stationary solution to the initial boundary value problem to this system, which has been recently extended to a more general case in a work by KAWASHIMA et al. [14].

Throughout this paper, we use the following:

Notations: Let $p$ be a constant such that $1 \leqq p \leqq \infty$, and $l$ be a non-negative integer. $\sigma \in(0,1)$ is a constant. We denote the norms of the usual Sobolev spaces $L^{p}\left(\mathbb{R}^{+}\right)$(with $p \neq 2$ and $p \geqq 1$ ) and $L^{2}\left(\mathbb{R}^{+}\right)$by $\|\cdot\|_{p}$ and $\|\cdot\|$, respectively. $\mathcal{B}^{l+\sigma}\left(\mathbb{R}^{+}\right)$denotes the Hölder space of continuous functions $f(x)$, defined in $\mathbb{R}^{+}$, which have the Hölder continuous derivatives with exponent $\sigma$ up to $l$ th order, and $\|\cdot\|_{l+\sigma}$ is its norm. $H^{l}\left(\mathbb{R}^{+}\right)$is the Sobolev space which is the set of $L^{2}\left(\mathbb{R}^{+}\right)$functions with the square integrable derivatives up to $l$ th order, and its norm is denoted by $\|\cdot\|_{H^{l}}$. Let $Q_{T}:=(0, t) \times \mathbb{R}^{+} . \mathcal{B}^{\sigma / 2, \sigma}\left(Q_{T}\right)$ denotes the Hölder space of continuous functions $u(t, x)$ which have the Hölder exponents $\sigma / 2$ and $\sigma$ with respect to $t$ and $x$, respectively. Its norm is $\|\cdot\|_{\sigma / 2, \sigma}\left(Q_{T}\right) \cdot \mathcal{B}^{1+\sigma / 2,1+\sigma}\left(Q_{T}\right)=$ $\left\{u \in \mathcal{B}^{\sigma / 2, \sigma}\left(Q_{T}\right): u_{t}, u_{x} \in \mathcal{B}^{\sigma / 2, \sigma}\left(Q_{T}\right)\right\},\|\cdot\|_{1+\sigma / 2,1+\sigma}\left(Q_{T}\right)$ denotes its norm, and $\mathcal{B}^{1+\sigma / 2,2+\sigma}\left(Q_{T}\right)=\left\{u \in \mathcal{B}^{\sigma / 2, \sigma}\left(Q_{T}\right): u_{t}, u_{x}, u_{x x} \in \mathcal{B}^{\sigma / 2, \sigma}\left(Q_{T}\right)\right\}$, $\|\cdot\|_{1+\sigma / 2,2+\sigma}\left(Q_{T}\right)$ denotes its norm. To simplify the notations, we shall denote the spaces $\mathcal{B}^{\sigma / 2, \sigma}\left(Q_{T}\right), \quad \mathcal{B}^{1+\sigma / 2,1+\sigma}\left(Q_{T}\right)$ and $\mathcal{B}^{1+\sigma / 2,2+\sigma}\left(Q_{T}\right)$ by $\mathcal{A}_{T}^{\sigma}, \mathcal{B}_{T}^{1+\sigma}$ and $\mathcal{C}_{T}^{2+\sigma}$, and their norms by $|\cdot|_{\sigma, T},\|\cdot\|_{1+\sigma, T}$ and $\||\cdot|\|_{2+\sigma, T}$, respectively. Let $X$ be a Banach space and $0<t_{1}<t_{2} \leqq \infty$. We use $C\left(\left[t_{1}, t_{2}\right] ; X\right)$ to denote the Banach space of continuous functions $u(t)$ on $\left[t_{1}, t_{2}\right]$ with values in $X$, and $L^{2}\left(t_{1}, t_{2} ; X\right)$ to denote the Banach space of square summable functions $u(t)$ on $\left[t_{1}, t_{2}\right]$ with values in $X$.

$C$ denotes the universal constant which is independent of $t$ and may vary from line to line. $\delta, \varepsilon$ are positive constants which can taken suitably small, we still denote $C \delta, C \varepsilon$ by $\delta, \varepsilon$, respectively.

Our main results are now stated below:

Theorem 1.1. Assume that $u_{b}, u_{*}$ and the infinite states satisfy that $u_{b}<0$, and that either $(i)$ subsonic case $(C)$, namely, $-\hat{C}\left(v_{+}\right)<u_{+}<0$ and $u_{*} \leqq u_{b}<u_{+}$, or (ii) $u_{+}>0$ and $u_{*} \leqq u_{b}<0$.

Suppose furthermore that $\rho_{0} \in \mathcal{B}^{1+\sigma}\left(\mathbb{R}^{+}\right), u_{0} \in \mathcal{B}^{2+\sigma}\left(\mathbb{R}^{+}\right)$for some $\sigma \in(0,1)$; and $\left(\rho_{0}-\rho_{+}, u_{0}-u_{+}\right) \in H^{1}\left(\mathbb{R}^{+}\right) \times H^{1}\left(\mathbb{R}^{+}\right)$, such that $\delta:=$ $\left|\rho_{+}-\rho_{-}\right|+\left|u_{+}-u_{b}\right|,\left\|\left(\rho_{0}-\rho_{+}, u_{0}-u_{+}\right)\right\|_{H^{1}}$ are suitably small. And the compatibility conditions of $0^{\text {th }}$ and $1^{\text {st }}$ order are satisfied.

Then there exists a unique classical global solution $(\rho, u)(t, x)$ to the problem (1.1)-(1.5), such that for any fixed $T>0$

$$
\begin{aligned}
& \rho \in \mathcal{B}_{T}^{1+\sigma}, u \in \mathcal{C}_{T}^{2+\sigma}, \quad \rho-\rho^{R}, u-u^{R} \in C\left([0, \infty) ; L^{2}\left(\mathbb{R}^{+}\right)\right) ; \\
& \rho_{x}, u_{x} \in C\left([0, \infty) ; L^{2}\left(\mathbb{R}^{+}\right)\right) \cap L^{2}\left(0, \infty ; L^{2}\left(\mathbb{R}^{+}\right)\right) ; \quad u_{x x} \in L^{2}\left(0, \infty ; L^{2}\left(\mathbb{R}^{+}\right)\right) .
\end{aligned}
$$

Moreover, we assert that as $t \rightarrow \infty$ the solution $(\rho, u)(t, x)$ converges to a rarefaction wave $\left(\rho^{R}, u^{R}\right)\left(\frac{x}{t}\right)$, that is

$$
\lim _{t \rightarrow \infty} \sup _{x \in \mathbb{R}^{+}}\left|(\rho, u)(t, x)-\left(\rho^{R}, u^{R}\right)\left(\frac{x}{t}\right)\right|=0 .
$$


Here, the functions $\rho_{0}^{R}=\rho_{0}^{R}(x), u_{0}^{R}=u_{0}^{R}(x)$ are defined by

$$
\rho_{0}^{R}(x)=\left\{\begin{array}{ll}
\rho_{+}, & \text {for } x>0 ; \\
\rho_{-}, & \text {for } x<0
\end{array} \quad \text { and } \quad u_{0}^{R}(x)= \begin{cases}u_{+}, & \text {for } x>0 \\
u_{b}, & \text { for } x<0\end{cases}\right.
$$

In the following context, we shall employ the standard continuation argument based on a local existence theorem and some a priori estimates (see Proposition 3.2) to prove this theorem. The main difficulties in our proof of Theorem 1.1 arise from the presence of the boundary condition and that we formulate the problem in the Eulerian coordinate. For our initial boundary value problem, the classification of time-asymptotic states is more complicated than the classification for the problem in the Lagrangian coordinate. There are non-linear waves which are called the boundary layer solution or the superposition of a rarefaction wave and a stationary solution. The latter case is considered in [30]. And there are only a few results on the stability of superposition waves, see, for example [16,17]. Since we consider the problem in the Eulerian coordinate, the system is more complicated than that in the Lagrangian mass coordinate which usually makes the form of equations simpler and the treatment of the equations easier. Matsumura and NishiHARA [26], HuANG et al. [4] transform the inflow problem in the Eulerian coordinate into that in the Lagrangian coordinate with a prescribed moving boundary, and prove partly the stability of the boundary layer solution, viscous shock wave, or the superposition of a rarefaction wave and a stationary solution. However, for our outflow problem, such a transformation results in a free boundary problem which makes the treatment of the boundary more difficult. To overcome this difficulty, we therefore employ a direct energy method to the reformulated problem for the Navier-Stokes equations in the Eulerian coordinate and take into account the dissipative effect of the boundary terms. Finally, it is more difficult to justify the formal argument on the derivation of the energy estimates for the higher derivatives of the unknown functions. For the details of a rigorous argument, we refer to KAWASHIMA et al. [10] and omit them in this paper.

The remaining part of this article is organized as follows: In Section 2, since rarefaction waves are not smooth, we must construct a smooth approximation of those rarefaction waves by using the Burgers equation, instead of the smooth approximation of the initial data as usual. Then we derive the time-decay rates for the smooth rarefaction waves by making use of the results by KAWASHIMA and TANAKA [12]. Next we reformulate the problem and restate our main theorem in Section 3. A proposition on the a priori estimates is formulated. For the reformulated initial boundary value problem we establish the energy estimates in Sections 4, and 5. By those estimates we prove that proposition at the end of Section 5. Finally, in Section 6, we shall prove the asymptotic stability of the solution.

\section{Construction and decay estimates of the smoothed rarefaction waves}

Since the rarefaction wave $\left(\rho^{R}, u^{R}\right)\left(\frac{x}{t}\right)$ is not smooth, we intend to construct a smooth approximation of the rarefaction wave $(\tilde{\rho}, \tilde{u})(t, x)$ in this section. Then we derive the estimates of decay rates for this smooth wave $(\tilde{\rho}, \tilde{u})(t, x)$. To this end, we 
make use of an approach which has been developed by KAWASHIMA and TANAKA [12], based on a parabolic approximation instead of the usual method based on the smooth approximation of initial data. For the sake of simplicity of notations, we still denote $(\tilde{\rho}, \tilde{u})(t, x)$ by $(\rho, u)(t, x)$ in the remaining part of this section, and change the notation $(\rho, u)(t, x)$ back to $(\tilde{\rho}, \tilde{u})(t, x)$ in the other Sections 4-6.

Let the function $w=w(t, x)$ be a solution to the problem for the Burgers equation

$$
w_{t}+w w_{x}=\kappa w_{x x}
$$

with initial data

$$
w(x, 0)=w_{ \pm} \text {for } x \geq 0,
$$

where $w_{ \pm}$are given by $w_{ \pm}:=u_{ \pm}+C\left(\rho_{ \pm}\right)$satisfying

$$
w_{+}>w_{-}
$$

We then define the functions

$$
\rho=\rho(t, x), \quad u=u(t, x)
$$

by the equations below

$$
\lambda(\rho, u):=u+C(\rho)=w(1+t, x), \quad \text { and } \quad \frac{\mathrm{d} u}{\mathrm{~d} \rho}=\frac{C(\rho)}{\rho} .
$$

for any $t, x \geqq 0$. Then we can easily see that $\rho, u$ are not independent functions, and one of these two functions can be written in terms of another one, that is

$$
\rho=\rho(u), \quad u=u(\rho) .
$$

There holds

$$
\begin{aligned}
& w_{t}=\lambda_{\rho} \rho_{t}=\lambda_{u} u_{t}, \\
& w_{x}=\lambda_{\rho} \rho_{x}=\lambda_{u} u_{x},
\end{aligned}
$$

and

$$
w_{x x}=\lambda_{\rho} \rho_{x x}+\lambda_{\rho \rho}\left(\rho_{x}\right)^{2}=\lambda_{u} u_{x x}+\lambda_{u u}\left(u_{x}\right)^{2} .
$$

Here we have used the notations:

$$
\lambda_{\rho}=\frac{\mathrm{d}}{\mathrm{d} \rho} \lambda(\rho, u(\rho)) \text { and } \lambda_{u}=\frac{\mathrm{d}}{\mathrm{d} u} \lambda(\rho(u), u) .
$$

It then follows from (2.1)-(2.7) that

$$
u_{x}=\frac{\mathrm{d} u}{\mathrm{~d} \rho} \rho_{x}=\frac{C(\rho)}{\rho} \rho_{x} .
$$


Next we are going to find the equations which are satisfied by $\rho, u$. There holds

$$
\begin{aligned}
\rho_{t}+(\rho u)_{x} & =\rho_{t}+u \rho_{x}+\rho u_{x}=\rho_{t}+(u+C(\rho)) \rho_{x} \\
& =\frac{1}{\lambda_{\rho}}\left(w_{t}+w w_{x}\right)=\frac{1}{\lambda_{\rho}} \kappa w_{x x} \\
& =\kappa \rho_{x x}+\kappa \frac{\lambda \rho \rho}{\lambda_{\rho}}\left(\rho_{x}\right)^{2}
\end{aligned}
$$

Similarly, one has

$$
\begin{aligned}
\rho\left(u_{t}+u u_{x}\right)+p(\rho)_{x} & =\rho\left(u_{t}+(u+C(\rho)) u_{x}\right)=\frac{\rho}{\lambda_{u}} \kappa w_{x x} \\
& =\kappa \rho\left(u_{x x}+\frac{\lambda_{u u}}{\lambda_{u}}\left(u_{x}\right)^{2}\right) .
\end{aligned}
$$

Furthermore, after straightforward calculations, one has

$$
\frac{\lambda_{\rho \rho}}{\lambda_{\rho}}=\frac{\gamma-3}{2 \rho}, \text { and } \quad \frac{\lambda_{u u}}{\lambda_{u}}=0
$$

which combined with (2.8) and (2.9) yields $\rho, u$ satisfies the following equations:

$$
\begin{aligned}
\rho_{t}+(\rho u)_{x} & =f, \\
\rho\left(u_{t}+u u_{x}\right)+p(\rho)_{x} & =g
\end{aligned}
$$

where $f, g$ are defined by

$$
\begin{aligned}
& f:=\kappa \rho_{x x}+\frac{(\gamma-3) \kappa}{2} \frac{\left(\rho_{x}\right)^{2}}{\rho}, \\
& g:=\kappa \rho u_{x x} .
\end{aligned}
$$

In what follows, we intend to derive the decay rates of the smoothed rarefaction waves $\rho, u$ in $L^{p}\left(\mathbb{R}^{+}\right)$by using the decay rates of $w$ which have been established by KAWASHIMA and TANAKA [12], and are restated in Lemma 2.1 below for the sake of an easy reference. Let

$$
\delta^{\prime}=w_{+}-w_{-}
$$

One can easily show that $C^{\prime} \delta \leqq \delta^{\prime} \leqq C \delta$ for two positive constants $C^{\prime}, C$ which are independent of $\delta, \delta^{\prime}$.

Lemma 2.1. For the solution $w(t, x)$ to (2.1) and (2.2), we have the following conclusions:

(i) $w(t, x)$ is a strictly increasing function of $x \in \mathbb{R}$ for each fixed $t>0$ and

$$
w_{+}>w(t, x)>w_{-} \text {for } x \in \mathbb{R} \text { and } t>0 \text {. }
$$


(ii) (The $L^{p}$-estimates) For $1<p \leqq \infty$, there holds

$$
\left\|w(t, \cdot)-w^{R}\left(\begin{array}{c}
\cdot \\
t
\end{array}\right)\right\|_{p} \leqq C(1+t)^{-\frac{1}{2}\left(1-\frac{1}{p}\right)} .
$$

And there holds for any $t>0$ and for any $1 \leqq p \leqq \infty$ that

$$
\left\|\partial_{x} w(t, \cdot)-\partial_{x} w^{R}\left(\frac{\cdot}{t}\right)\right\|_{p} \leqq C(1+t)^{-\frac{1}{2 p}} t^{-\left(1-\frac{1}{p}\right)} \leqq C t^{-\left(1-\frac{1}{2 p}\right)} .
$$

(iii) (The derivatives' estimates) For $1 \leqq p \leqq \infty$, we have the following decay estimates for the derivatives of the solution $w(t, x)$ :

$$
\begin{aligned}
&\left\|\partial_{x} w(t, \cdot)\right\|_{p} \leqq C \delta^{\prime} \frac{1}{p} t^{-\left(1-\frac{1}{p}\right)}, \quad t>0 \\
&\left\|\partial_{x}^{k} w(t, \cdot)\right\|_{p} \leqq C \delta^{\prime} t^{-\frac{1}{2}\left(k-\frac{1}{p}\right)}, \quad k=1,2,3, \ldots, t>0 \\
&\left\|\partial_{x}^{k} w(t, \cdot)\right\|_{p} \leqq C(1+t)^{-\frac{1}{2}} t^{-\frac{1}{2}\left(k-\frac{1}{p}\right)} \leqq C t^{-\frac{1}{2}\left(k+1-\frac{1}{p}\right)}, \quad k=2,3, \ldots, t>0 .
\end{aligned}
$$

(iv) Let $y_{ \pm}:=\left(x-w_{ \pm} t\right) / \sqrt{2 t}, \quad \Omega_{+}:=\left\{x \geqq w_{ \pm} t\right\}, \quad \Omega_{-}:=\left\{x \leqq w_{ \pm} t\right\}$. We have

(1) If $(t, x) \in \Omega_{+}$, then $y_{+} \geqq 0$ and

$$
\begin{aligned}
& \left|w(t, x)-w_{+}\right| \leqq C \delta^{\prime}\left(1+\delta^{\prime} \sqrt{t}\right)^{-1} \exp \left(-y_{+}^{2}\right), \\
& \left|\partial_{x}^{k} w(t, x)\right| \leqq C \delta^{\prime} t^{-\frac{k}{2}}\left(1+\delta^{\prime} \sqrt{t}\right)^{-1}\left(1+y_{+}\right)^{k} \exp \left(-y_{+}^{2}\right), \quad \forall t>0 ;
\end{aligned}
$$

(2) If $(t, x) \in \Omega_{-}$, then there holds $y_{-} \leqq 0$ and

$$
\begin{aligned}
& \left|w(t, x)-w_{-}\right| \leqq C \delta^{\prime}\left(1+\delta^{\prime} \sqrt{t}\right)^{-1} \exp \left(-y_{-}^{2}\right), \\
& \left|\partial_{x}^{k} w(t, x)\right| \leqq C \delta^{\prime} t^{-\frac{k}{2}}\left(1+\delta^{\prime} \sqrt{t}\right)^{-1}\left(1-y_{-}\right)^{k} \exp \left(-y_{-}^{2}\right), \quad \forall t>0 .
\end{aligned}
$$

(2a) Especially, if $w_{-}>0$, then we conclude that the point $(t, x):=(t, 0) \in$ $\Omega_{-}$; thus

$$
\begin{aligned}
\left|w(t, 0)-w_{-}\right| \leqq & C \delta^{\prime}\left(1+\delta^{\prime} \sqrt{t}\right)^{-1} \exp \left(-\frac{w_{-}^{2} t}{2}\right) \\
\left|\partial_{x}^{k} w(t, x)\right| \leqq & C \delta^{\prime} t^{-\frac{k}{2}}\left(1+\delta^{\prime} \sqrt{t}\right)^{-1}\left(1+w_{-} \sqrt{\frac{t}{2}}\right)^{k} \\
& \exp \left(-\frac{w_{-}^{2} t}{2}\right), \quad \forall t>0 .
\end{aligned}
$$


Employing the above decay estimates on the function $w$, we can easily obtain the corresponding time decay estimates of the smooth rarefaction waves $\rho$ and $u$. By the definition, we can find that the functions $\rho(t, x)$ and $u(t, x)$ depend only on the value of $w(t, x)$ with $t \geqq 1$. So, they have no singularity at $t=0$ though $w(t, x)$ has possible singular there.

Lemma 2.2. Assume that $w(t, x)$ is a solution to (2.1) and (2.2), we can obtain by solving (2.4) the corresponding smooth rarefaction waves $\rho, u$, which satisfy the following estimates

(i) $\rho, u$ are strictly increasing functions of $x \in \mathbb{R}$ for each fixed $t>0$ and

$$
\begin{aligned}
& \rho_{x}(t, x)>0, \quad u_{x}(t, x)>0, \\
& \rho_{+}>\rho(t, x)>\rho_{-}, \text {and } u_{+}>u(t, x)>u_{-} \text {for } x \in \mathbb{R} \text { and } t>0 .
\end{aligned}
$$

(ii) (The $L^{p}$-estimates) For $1<p \leqq \infty$, there holds

$$
\left\|(\rho, u)(t, \cdot)-\left(\rho^{R}, u^{R}\right)\left(\frac{\cdot}{1+t}\right)\right\|_{p} \leqq C(1+t)^{-\frac{1}{2}\left(1-\frac{1}{p}\right)} .
$$

And for $1 \leqq p \leqq \infty$, we have

$$
\left\|\partial_{x}(\rho, u)(t, \cdot)-\partial_{x}\left(\rho^{R}, u^{R}\right)\left(\frac{\cdot}{1+t}\right)\right\|_{p} \leqq C(1+t)^{-\left(1-\frac{1}{2 p}\right)} .
$$

(iii) (The derivatives' estimates) For $1 \leqq p \leqq \infty$, we have the following decay estimates for the derivatives of the solution $(\rho, u)$ :

$$
\begin{aligned}
&\left\|\partial_{x}(\rho, u)(t, \cdot)\right\|_{p} \leqq C \delta^{\frac{1}{p}}(1+t)^{-\left(1-\frac{1}{p}\right)}, \\
&\left\|\partial_{x}^{k}(\rho, u)(t, \cdot)\right\|_{p} \leqq C \delta(1+t)^{-\frac{1}{2}\left(k-\frac{1}{p}\right)}, \quad k=1,2,3, \ldots, \\
&\left\|\partial_{x}^{k}(\rho, u)(t, \cdot)\right\|_{p} \leqq C(1+t)^{-\frac{1}{2}\left(k+1-\frac{1}{p}\right)}, \quad k=2,3, \ldots
\end{aligned}
$$

(iv) (The boundary estimate) For the boundary value, we have

$$
\begin{aligned}
& |\psi(t, 0)| \leqq C \delta(1+\delta \sqrt{t})^{-1} \exp \left(-\frac{C t}{2}\right), \\
& \left|u_{t}(t, 0)\right| \leqq C \delta(1+\delta \sqrt{t})^{-1} \exp \left(-\frac{C t}{2}\right) .
\end{aligned}
$$

Here, $C$ is a positive constant and the function $\psi:=u-\tilde{u}$ which will be defined in Section 3, and $u$ is the solution to the initial boundary value problem, while $\tilde{u}$ is just $u$ here, the smoothed rarefaction wave.

Proof. By the definition of $\rho, u$ one can find that the proofs of items (i)-(iii) are easy. It needs only to prove (iv). First of all, we show that $w_{-}>0$. From both Figs. $\mathrm{C}$ and $\mathrm{D}$ in the state space $v-u$ for the rarefaction cases, we can determine the value of $\rho_{-}$since we choose the point $\left(v_{-}, u_{b}\right)$ at the curve $R_{2}$ and $u_{b}$ is given. Then by the definition of $w$ one has $w_{-}=u_{-}+C\left(\rho_{-}\right)>0$. Next, applying conclusion (iv) in Lemma 2.1 and estimate (2.1), we assert that (iv) of this lemma is valid. 
We shall see in the sequel that the boundary values exponentially decay and these decay estimates are crucial for the proof of our main theorem. And the proof is based on the $L^{2}$-energy method, we shall deal with the terms arising from the boundary.

\section{Reformulation of the problem}

In this section, we make use of the smoothed approximation rarefaction wave $(\rho, u)$ which has been constructed in Section 2, and we denote it hereafter by $(\tilde{\rho}, \tilde{u})$ and $(\rho, u)$ is left to denote the solution to the problem (1.1)-(1.5). Keeping it in mind, we proceed to reformulate the problem under consideration. We define

$$
\phi(t, x):=\rho(t, x)-\tilde{\rho}(t, x), \quad \psi(t, x):=u(t, x)-\tilde{u}(t, x) .
$$

Then it is easy to check that $(\phi, \psi)$ satisfies

$$
\begin{aligned}
\phi_{t}+u \phi_{x}+\rho \psi_{x} & =F, \\
\rho\left(\psi_{t}+u \psi_{x}\right)+p^{\prime}(\rho) \phi_{x}-\mu \psi_{x x} & =G,
\end{aligned}
$$

where $F, G$ are defined by

$$
\begin{aligned}
F & :=-\left(\kappa \tilde{\rho}_{x x}+\frac{(\gamma-3) \kappa}{2} \frac{\tilde{\rho}_{x}^{2}}{\tilde{\rho}}+\tilde{u}_{x} \phi+\psi \tilde{\rho}_{x}\right), \\
G & :=\left(\phi \frac{p^{\prime}(\tilde{\rho})}{\tilde{\rho}}-\left(p^{\prime}(\rho)-p^{\prime}(\tilde{\rho})\right)\right) \tilde{\rho}_{x}-\rho \psi \tilde{u}_{x}+(\mu-\kappa \rho) \tilde{u}_{x x} .
\end{aligned}
$$

The boundary and initial conditions turn now out to be

$$
\begin{aligned}
\psi(t, 0) & =u_{b}-\tilde{u}(t, 0), \\
\phi(0, x)=\rho_{0}(x)-\tilde{\rho}_{0}(x), \quad \psi(0, x) & =u_{0}(x)-\tilde{u}_{0}(x) .
\end{aligned}
$$

Moreover, for suitably small $\delta$ and $\phi, \psi$ (It can be confirmed in the following context that $\phi, \psi$ are small), we have

$$
|F| \leqq C\left(\left|\tilde{\rho}_{x x}\right|+\left|\tilde{\rho}_{x}\right|^{2}+\left|\tilde{\rho}_{x} \psi\right|+\left|\phi \tilde{u}_{x}\right|\right)
$$

and

$$
|G| \leqq C\left(\left|\phi \tilde{\rho}_{x}\right|+\left|\psi \tilde{u}_{x}\right|+\left|\tilde{u}_{x x}\right|\right)
$$

Therefore, we can now restate our main results as follows:

Theorem 3.1. Assume that $u_{b}<0$, and that either $(i)$ subsonic case $(C),-\hat{C}\left(v_{+}\right)<$ $u_{+}<0$ and $u_{*} \leqq u_{b}<u_{+}$, or (ii) $u_{+}>0$ and $u_{*} \leqq u_{b}<0$.

Suppose further that $\phi_{0} \in \mathcal{B}^{1+\sigma}\left(\mathbb{R}^{+}\right), \psi_{0} \in \mathcal{B}^{2+\sigma}\left(\mathbb{R}^{+}\right)$and $\left(\phi_{0}, \psi_{0}\right) \in$ $H^{1}\left(\mathbb{R}^{+}\right) \times H^{1}\left(\mathbb{R}^{+}\right)$, such that $\delta:=\left|\rho_{+}-\rho_{-}\right|+\left|u_{+}-u_{b}\right|$ and $\left\|\left(\phi_{0}, \psi_{0}\right)\right\|_{H^{1}}$ are suitably small. And the compatibility conditions of Oth and 1 st order are satisfied. 
Then there exists a unique global solution $(\phi, \psi)(t, x)$ to the problem (3.2)(3.7), such that

$$
\begin{aligned}
& \phi \in \mathcal{B}_{T}^{1+\sigma}, \quad \psi \in \mathcal{C}_{T}^{2+\sigma}, \quad \phi, \psi \in C\left([0, \infty) ; H^{1}\right) \\
& \phi_{x} \in L^{2}\left(0, \infty ; L^{2}\right) ; \quad \psi_{x} \in L^{2}\left(0, \infty ; H^{1}\right) .
\end{aligned}
$$

Moreover, as time goes to infinity the solution $(\phi, \psi)(t, x)$ converges in $L^{\infty}$-norm to $(0,0)$, namely

$$
\sup _{x \in \mathbb{R}^{+}}|(\phi, \psi)(t, x)| \rightarrow 0, \text { as } t \rightarrow \infty
$$

In order to prove Theorem 3.1, we make use of the standard method, which is based on a local existence theorem and a priori estimates. The local existence theorem is similar to that in the previous paper by the authors [10]. On the other hand, the a priori estimates are collected in Proposition 3.2.

To simplify the notations we define

$$
M(t)^{2}:=\int_{0}^{t}\left(\left\|\phi_{x}(\tau)\right\|^{2}+\left\|\psi_{x}(\tau)\right\|_{H^{1}}^{2}+|\phi(\tau, 0)|^{2}+\left|\phi_{x}(\tau, 0)\right|^{2}\right) \mathrm{d} \tau
$$

and

$$
N(t):=\sup _{0 \leqq \tau \leqq t}\|(\phi, \psi)(\tau)\|_{H^{1}\left(\mathbb{R}^{+}\right)} \leqq E_{0}
$$

and $E_{0}$ is suitably small so that $\rho=\phi+\tilde{\rho} \geqq \rho_{-}-C_{1} E_{0} \geqq \frac{1}{2} \rho_{-}$. Here $C_{1}$ is a constant in the inequality $\|\phi\|_{L^{\infty}} \leqq C_{1}\|\phi\|_{H^{1}}$.

We now state the a priori estimates:

Proposition 3.2. (A priori estimates) Assume that ( $\phi, \psi)$ is a solution to (3.2)-(3.9) satisfying

$$
\begin{aligned}
& \phi \in C\left([0, T] ; H^{1}\right) \cap \mathcal{B}_{T}^{1+\sigma}, \quad \psi \in C\left([0, T] ; H^{1}\right) \cap \mathcal{C}_{T}^{2+\sigma} ; \\
& \inf _{Q_{T}} \rho(t, x)>0,
\end{aligned}
$$

for any fixed $T>0$. Then there exists a constant $\theta \in\left(0, \frac{1}{2}\right)$ and a suitably small constant $\varepsilon_{0}>0$, such that if $N(t)+\delta \leqq \varepsilon_{0}$, then the following estimates hold

$$
\begin{aligned}
& \|(\phi, \psi)(t)\|_{H^{1}}^{2}+\int_{0}^{t}\left(\left\|\phi_{x}(\tau)\right\|^{2}+\left|\phi, \phi_{x}\right|^{2}(\tau, 0)+\left\|\psi_{x}(\tau)\right\|_{H^{1}}^{2}\right) \mathrm{d} \tau \\
& \quad \leqq C\left(\delta^{2 \theta}+\left\|\left(\phi_{0}, \psi_{0}\right)\right\|_{H^{1}}^{2}\right)
\end{aligned}
$$

for all $t \geqq 0$. Here $\varepsilon_{0}, C$ are independent of $t, \delta$. 
Proof. We divide the proof of this proposition into three steps, each is stated as a lemma, namely Lemmas 4.1, 4.2 and 4.3. Then combining these three lemmas we complete the proof of Proposition 3.2. Since we have carried out a rigorous argument in [10] to derive the norms of the derivatives of the unknown functions (for example the term $\left\|\phi_{x}\right\|$ in Section 5) for the case of the stationary solution, and a rigorous argument for the present case is similar to that, we omit here the details of that procedure and state only the following formal argument in this regard.

Step 1. The first step is the following basic energy estimate stated in Lemma 4.1 in the next section.

\section{The first energy estimates}

From now on, to derive the energy estimates, we assume that $(\phi, \psi)$ is a solution to (3.2)-(3.9) which satisfies the properties (3.12) and (3.13).

First of all, in this section, we shall establish the first energy estimate. To this end, as in [10], we define an energy function by

$$
\rho \mathcal{E}=\rho\left\{\frac{1}{2}(u-\tilde{u})^{2}+\Psi(\rho, \tilde{\rho})\right\}=\rho\left\{\frac{1}{2} \psi^{2}+\Psi(\rho, \tilde{\rho})\right\},
$$

where

$$
\Psi(\rho, \tilde{\rho})=\int_{\tilde{\rho}}^{\rho} \frac{p(\eta)-p(\tilde{\rho})}{\eta^{2}} \mathrm{~d} \eta .
$$

After a straightforward computation, we find that $\mathcal{E}$ satisfies

$$
\begin{aligned}
(\rho \mathcal{E})_{t}+\{(\rho u \mathcal{E})+(p-\tilde{p}) \psi\}_{x}-\left\{\mu \psi \psi_{x}\right\}_{x} \\
\quad=-\mu\left|\psi_{x}\right|^{2}-\left\{\rho \psi^{2}+p(\rho)-p(\tilde{\rho})-p^{\prime}(\tilde{\rho}) \phi\right\} \tilde{u}_{x}+R \\
R:=-\frac{\kappa}{\tilde{\rho}} p^{\prime}(\tilde{\rho}) \phi\left(\tilde{\rho}_{x x}+\frac{\gamma-3}{2}\left(\tilde{\rho}_{x}\right)^{2} \tilde{\rho}^{-1}\right)+\psi \tilde{u}_{x x}(\mu-\kappa \rho) .
\end{aligned}
$$

Here $R$ can be estimated as

$$
|R| \leqq C\left(|\phi|\left(\left|\tilde{\rho}_{x x}\right|+\left(\tilde{\rho}_{x}\right)^{2}\right)+\left|\psi \tilde{u}_{x x}\right|\right) .
$$

Now we can state the first energy estimate as

Lemma 4.1. Let $\theta \in\left(0, \frac{1}{2}\right)$ be the same constant as in Proposition 3.2. Then there is a positive constant $\varepsilon_{1}$ such that if $N(t)+\delta \leqq \varepsilon_{1}$, then the following estimate holds for any $t \geqq 0$

$$
\begin{aligned}
& \|(\phi, \psi)(t)\|^{2}+\int_{0}^{t}\left\{\left\|\psi_{x}(\tau)\right\|^{2}+|(\phi, \psi)(\tau, 0)|^{2}\right\} \mathrm{d} \tau \\
& \leqq C\left(\left\|\left(\phi_{0}, \psi_{0}\right)\right\|^{2}+\delta^{\theta}(N(t)+M(t))\right) .
\end{aligned}
$$

Here $\varepsilon_{1}, C$ are independent of $t, \delta$. 
Proof. Integrating Equation (4.3) with respect to $(t, x)$ over $(0, t) \times(0, \infty)$ yields

$$
\begin{aligned}
& \int_{0}^{\infty} \rho \mathcal{E} \mathrm{d} x+\mu \int_{0}^{t}\left(\left\|\psi_{x}\right\|^{2}+C \int_{0}^{\infty}\left(\rho \psi^{2}+\phi^{2}\right) \tilde{u}_{x} \mathrm{~d} x\right) \mathrm{d} \tau \\
& \quad-\left.\int_{0}^{t}\left(\rho u \mathcal{E}+(p-\tilde{p}) \psi-\mu \psi \psi_{x}\right)\right|_{x=0} \mathrm{~d} \tau \\
& \leqq \int_{0}^{\infty} \rho_{0} \mathcal{E}_{0} \mathrm{~d} x+C \int_{0}^{t} \int_{0}^{\infty}\left(|\phi|\left(\left|\tilde{\rho}_{x x}\right|+\left(\tilde{\rho}_{x}\right)^{2}\right)+\left|\psi \tilde{u}_{x x}\right|\right) \mathrm{d} x \mathrm{~d} \tau .
\end{aligned}
$$

Here, the fact that $\tilde{u}_{x}>0$ has been used. We now handle the boundary terms and the right-hand side terms in (4.6) as follows. From the relation

$$
-\left.(\rho u \mathcal{E}+(p-\tilde{p}) \psi)\right|_{x=0}=-\left.\rho u\left\{\frac{1}{2} \psi^{2}+\Psi(\rho, \tilde{\rho})\right\}\right|_{x=0}-\left.(p-\tilde{p}) \psi\right|_{x=0},
$$

recalling $u_{b}<0$, we have

$$
\begin{aligned}
\int_{0}^{t}-\left.\rho u\left\{\frac{1}{2} \psi^{2}+\Psi(\rho, \tilde{\rho})\right\}\right|_{x=0} \mathrm{~d} \tau & \geqq C \int_{0}^{t} \rho_{-}\left(-u_{b}\left(\phi(\tau, 0)^{2}+\psi(\tau, 0)^{2}\right)\right) \mathrm{d} \tau \\
& \geqq C^{\prime} \int_{0}^{t}|(\phi, \psi)(\tau, 0)|^{2} \mathrm{~d} \tau
\end{aligned}
$$

Using the mean value theorem, the Young inequality and the boundary estimate in Lemma 2.2, we obtain

$$
\begin{aligned}
\int_{0}^{t}|(p-\tilde{p}) \psi|_{x=0} \mid \mathrm{d} \tau & =\int_{0}^{t}\left|p^{\prime}(\eta)(\phi \psi)\right|_{x=0} \mid \mathrm{d} \tau \\
& \leqq C\left(\int_{0}^{t}|\phi(\tau, 0)|^{2} \mathrm{~d} \tau\right)^{\frac{1}{2}}\left(\int_{0}^{t}|\psi(\tau, 0)|^{2} \mathrm{~d} \tau\right)^{\frac{1}{2}} \\
& \leqq C M(t)\left(\int_{0}^{t} \delta^{2} \mathrm{e}^{-2 C \tau} \mathrm{d} \tau\right)^{\frac{1}{2}} \leqq C \delta M(t)
\end{aligned}
$$

As for the term on $\psi$, making use of the Sobolev embedding theorem, the Hölder inequality and Lemma 2.2, we have

$$
\begin{aligned}
& \left|\mu \int_{0}^{t}\left(\psi \psi_{x}\right)\right|_{x=0} \mathrm{~d} \tau\left|\leqq C \int_{0}^{t}\left\|\psi_{x}\right\|_{H^{1}}\right| \psi(\tau, 0) \mid \mathrm{d} \tau \\
& \quad \leqq C\left(\int_{0}^{t}\left\|\psi_{x}\right\|_{H^{1}}^{2} \mathrm{~d} \tau\right)^{\frac{1}{2}}\left(\int_{0}^{t}|\psi(\tau, 0)|^{2} d \tau\right)^{\frac{1}{2}} \leqq C \delta M(t) .
\end{aligned}
$$

For the remaining terms, making use of the Hölder inequality, then by combining (4.6)-(4.10), we arrive at

$$
\begin{aligned}
& \left.\int_{0}^{\infty} \rho \mathcal{E} \mathrm{d} x\right|_{0} ^{t}+\int_{0}^{t}\left(\mu\left\|\psi_{x}\right\|^{2}+C \int_{0}^{\infty}\left(\rho \psi^{2}+\phi^{2}\right) \tilde{u}_{x} \mathrm{~d} x+C^{\prime}|(\phi, \psi)(\tau, 0)|^{2}\right) \mathrm{d} \tau \\
& \leqq C \int_{0}^{t}\left(\|\phi\|\left(\left\|\tilde{\rho}_{x x}\right\|+\left\|\tilde{\rho}_{x}\right\|_{4}^{2}\right)+\|\psi\|\left\|\tilde{u}_{x x}\right\|\right) \mathrm{d} \tau+C \delta M(t) \\
& \leqq C \sup _{0 \leqq \tau}(\|\phi(\tau)\|+\|\psi(\tau)\|) \int_{0}^{t}\left(\left\|(\tilde{\rho}, \tilde{u})_{x x}\right\|+\left\|\tilde{\rho}_{x}\right\|_{4}^{2}\right) \mathrm{d} \tau+C \delta M(t)
\end{aligned}
$$


Invoking the decay estimates on the smooth solution $(\tilde{\rho}, \tilde{u})$, we have for any $t>0$

$$
\left\|\tilde{\rho}_{x x}\right\| \leqq C \delta(1+t)^{-\frac{3}{4}}, \quad\left\|\tilde{\rho}_{x x}\right\| \leqq C(1+t)^{-\frac{5}{4}}
$$

Therefore, a combination of these two decay rate estimates yields

$$
\left\|\tilde{\rho}_{x x}\right\| \leqq C \delta^{\theta}(1+t)^{-\frac{5-2 \theta}{4}},
$$

for any $0 \leqq \theta \leqq 1$. Here we have used the basic fact that if $0<C \leqq A, C \leqq B$, then $C \leqq \bar{A}^{\theta} B^{\overline{1}-\theta}$ for any $0 \leqq \theta \leqq 1$.

It then follows from Lemma 2.2, (4.11) and estimate (4.12) that

$$
\begin{aligned}
& \int_{0}^{\infty} \rho \mathcal{E} \mathrm{d} x+\int_{0}^{t}\left\{\mu\left\|\psi_{x}\right\|^{2}+C \int_{0}^{\infty}\left(\left(\rho \psi^{2}+\phi^{2}\right) \tilde{u}_{x} \mathrm{~d} x+|(\phi, \psi)(\tau, 0)|^{2}\right)\right\} \mathrm{d} \tau \\
& \quad \leqq C\left\|\left(\phi_{0}, \psi_{0}\right)\right\|^{2}+C N(t) \int_{0}^{t}\left(\delta^{\theta}(1+\tau)^{-\frac{5-2 \theta}{4}}+\delta^{\frac{1}{2}}(1+\tau)^{-\frac{3}{4} \times 2}\right) d \tau+C \delta M(t) \\
& \quad \leqq C\left(\left\|\left(\phi_{0}, \psi_{0}\right)\right\|^{2}+\delta^{\theta} N(t)+\delta M(t)\right) \\
& \leqq C\left(\left\|\left(\phi_{0}, \psi_{0}\right)\right\|^{2}+\delta^{\theta}(N(t)+M(t))\right)
\end{aligned}
$$

Here we have chosen $\delta<1$ and $\theta \in\left(0, \frac{1}{2}\right)$ so that $\frac{5-2 \theta}{4}>1$.

Remembering the fact that $\rho \geqq C$, we can easily obtain

$$
\int_{0}^{\infty} \rho \mathcal{E} \mathrm{d} x \geqq C\left(\|\phi\|^{2}+\|\psi\|^{2}\right) .
$$

Thus the proof of this Lemma is complete.

\section{The second energy estimates}

With the first energy estimate in hand, we now proceed to establish the second energy estimate. The main results are the following two lemmas, that is Lemmas 5.1 and 5.2.

Step 2. The second step aims at the proof of the following Lemma.

Lemma 5.1. There exists a positive constant $\varepsilon_{2} \leqq \varepsilon_{1}$ such that if $N(t)+\delta \leqq \varepsilon_{2}$, then the following estimate holds for any $t \geqq 0$

$$
\begin{aligned}
& \left\|\phi_{x}(t)\right\|^{2}+\int_{0}^{t}\left(\left\|\phi_{x}(\tau)\right\|^{2}+\left|\phi_{x}(\tau, 0)\right|^{2}\right) \mathrm{d} \tau \\
& \quad \leqq C\left\|\left(\phi_{0}, \psi_{0}\right)\right\|_{H^{1}}^{2}+C\left((N(t)+\delta) M(t)^{2}+\delta^{\theta}(M(t)+N(t))+\delta^{2}\right) .
\end{aligned}
$$

where $\varepsilon_{2}, C$ are independent of $t, \delta$. 
Proof. The proof of this lemma consists of two steps.

Firstly, we shall show that the function $\phi_{x}$ can estimated in terms of $N(t)$ and $M(t)$. To this end, we differentiate (3.2) formally with respect to $x$ to get

$$
\phi_{x t}+u \phi_{x x}+\rho \psi_{x x}=F_{x}-\tilde{u}_{x} \phi_{x}-\tilde{\rho}_{x} \psi_{x}-2 \phi_{x} \psi_{x}=: F_{1}
$$

Let us stop here to give a remark

Remark 5.1. The above computation is formal since there is a term $\phi_{x t}$ in (5.2). For the justification of such formal calculation, we refer to [10].

We now turn to the proof of Lemma 5.1. To simply the calculation in the second step below, we now transform the above equation of $\phi_{x}$ into that in the terms of $\frac{\phi_{x}}{\rho}$. To this end, we divide (5.2) by $\rho$ to get

$$
\frac{\phi_{x t}}{\rho}+u \frac{\phi_{x x}}{\rho}+\psi_{x x}=\frac{F_{1}}{\rho} .
$$

By the similar argument as in [10], we have

$$
\begin{aligned}
\left(\frac{\phi_{x}}{\rho}\right)_{t}+u\left(\frac{\phi_{x}}{\rho}\right)_{x}+\psi_{x x} & =\frac{F_{1}}{\rho}-\frac{\phi_{x}}{\rho^{2}}\left(\tilde{\rho}_{t}+\tilde{\rho}_{x} u+\phi_{t}+u \phi_{x}\right) \\
& =\frac{F_{1}}{\rho}+\frac{\phi_{x} u_{x}}{\rho}=: F_{2}
\end{aligned}
$$

Moreover,

$$
\left|F_{2}\right| \leqq C\left(\left|F_{1}\right|+\left|\phi_{x}\right|\left(\left|\psi_{x}\right|+\left|\tilde{u}_{x}\right|\right)\right)
$$

and by the definition of $F_{1}$, we obtain

$$
\begin{aligned}
\left|F_{1}\right| \leqq & C\left(\left|\tilde{\rho}_{x x x}\right|+\left|\tilde{\rho}_{x x} \tilde{\rho}_{x}\right|+\left|\tilde{\rho}_{x}\right|^{3}+\left|\phi \tilde{u}_{x x}\right|+\left|\psi \tilde{\rho}_{x x}\right|+\left|\phi_{x} \tilde{u}_{x}\right|\right. \\
& \left.+\left|\psi_{x} \tilde{\rho}_{x}\right|+\left|\phi_{x} \psi_{x}\right|\right) .
\end{aligned}
$$

Then multiplying (5.4) by $\frac{\phi_{x}}{\rho}$, integrating it with respect to $x$ and integration by parts, we obtain

$$
\frac{1}{2} \frac{\mathrm{d}}{\mathrm{d} t}\left\|\frac{\phi_{x}}{\rho}\right\|^{2}-\int_{0}^{\infty} u_{x}\left(\frac{\phi_{x}}{\rho}\right)^{2} \mathrm{~d} x-\left.\frac{1}{2} u\left(\frac{\phi_{x}}{\rho}\right)^{2}\right|_{x=0}+\int_{0}^{\infty} \frac{\psi_{x x} \phi_{x}}{\rho} \mathrm{d} x=\int_{0}^{\infty} \frac{F_{2} \phi_{x}}{\rho} \mathrm{d} x .
$$

Secondly, we try to remove the term of $\psi_{x x}$ in (5.7). To this end, we multiply (3.3) by $\frac{\phi_{x}}{\rho}$, and integrate the resulting equation with respect to $x$ to get

$$
\left(\psi_{t}+u \psi_{x}, \phi_{x}\right)+\int_{0}^{\infty}\left(p^{\prime}(\rho) \phi_{x}-\mu \psi_{x x}\right) \frac{\phi_{x}}{\rho} \mathrm{d} x=\int_{0}^{\infty} \frac{G \phi_{x}}{\rho} \mathrm{d} x .
$$


Here, we have used the notation $(f, g)$ to denote the inner product of two functions $f, g$. On the other hand, we have

$$
\begin{aligned}
\left(\psi_{t}, \phi_{x}\right) & =\frac{\mathrm{d}}{\mathrm{d} t}\left(\psi, \phi_{x}\right)-\left(\phi_{x t}, \psi\right) \\
& =\frac{\mathrm{d}}{\mathrm{d} t}\left(\psi, \phi_{x}\right)+\left.\left(\psi \phi_{t}\right)\right|_{x=0}+\left(\psi_{x}, \phi_{t}\right) .
\end{aligned}
$$

Then multiplying (5.7) by $\mu$, adding it to (5.8) and integrating the resultant with respect to $t$ yields

$$
\begin{aligned}
& \frac{\mu}{2}\left\|\frac{\phi_{x}}{\rho}\right\|^{2}+\left(\psi, \phi_{x}\right)-\mu \int_{0}^{t} \int_{0}^{\infty} u_{x}\left(\frac{\phi_{x}}{\rho}\right)^{2} \mathrm{~d} x \mathrm{~d} \tau-\left.\frac{\mu}{2} \int_{0}^{t} u\left(\frac{\phi_{x}}{\rho}\right)^{2}\right|_{x=0} \mathrm{~d} \tau \\
& \quad+\int_{0}^{t}\left(\left.\left(\psi \phi_{t}\right)\right|_{x=0}+\left(\psi_{x}, \phi_{t}\right)\right) \mathrm{d} \tau+\int_{0}^{t}\left(u \psi_{x}, \phi_{x}\right) \mathrm{d} \tau+\int_{0}^{t} \int_{0}^{\infty} \frac{p^{\prime}(\rho)}{\rho}\left|\phi_{x}\right|^{2} \mathrm{~d} x \mathrm{~d} \tau \\
& =\frac{\mu}{2}\left\|\frac{\phi_{0 x}}{\rho_{0}}\right\|^{2}+\left(\psi_{0}, \phi_{0 x}\right)+\int_{0}^{t} \int_{0}^{\infty} \frac{\left(G+F_{2}\right) \phi_{x}}{\rho} \mathrm{d} x \mathrm{~d} \tau
\end{aligned}
$$

We now estimate (5.10) as follows. Firstly, it is easy to estimate that

$$
\left|\left(\psi, \phi_{x}\right)\right| \leqq C_{\varepsilon}\|\psi\|^{2}+\varepsilon\left\|\phi_{x}\right\|^{2} .
$$

By the decay estimates in Lemma 2.2, we have

$$
\begin{aligned}
\left|\int_{0}^{t} \int_{0}^{\infty}\left(\psi_{x}+\tilde{u}_{x}\right)\left(\frac{\phi_{x}}{\rho}\right)^{2} \mathrm{~d} x \mathrm{~d} \tau\right| & \leqq C \int_{0}^{t}\left(\left\|\psi_{x}\right\|_{\infty}+\left\|\tilde{u}_{x}\right\|_{\infty}\right)\left\|\phi_{x}\right\|^{2} \mathrm{~d} \tau \\
& \leqq C \int_{0}^{t}\left(\left\|\psi_{x}\right\|_{H^{1}}+\delta\right)\left\|\phi_{x}\right\|^{2} \mathrm{~d} \tau \\
& \leqq C(N(t)+\delta) M(t)^{2}
\end{aligned}
$$

For the boundary term, we make use of (3.2)

$$
\left|\phi_{t}(t, x)\right| \leqq C\left(\left|\psi_{x}\right|+\left|\phi_{x}\right|+\left|\tilde{\rho}_{x x}\right|+\left|\tilde{\rho}_{x}\right|^{2}+\left|\phi \tilde{u}_{x}\right|+\left|\psi \tilde{\rho}_{x}\right|\right),
$$

then apply the Young inequality, Lemma 4.1, and the estimates (2.31) and (5.13) with $x=0$, to get

$$
\begin{aligned}
\left|\int_{0}^{t}\left(\psi \phi_{t}\right)(\tau, 0) \mathrm{d} \tau\right| \leqq & C \int_{0}^{t}|\psi(\tau, 0)|\left(\left|\phi_{x}(\tau, 0)\right|+\left|\psi_{x}(\tau, 0)\right|\right) \mathrm{d} \tau+C \delta^{2} \\
& +C \int_{0}^{t}|\psi(\tau, 0)|\left(|\phi(\tau, 0)|\left\|\tilde{u}_{x}\right\|_{\infty}+|\psi(\tau, 0)|\left\|\tilde{\rho}_{x}\right\|_{\infty}\right) \mathrm{d} \tau \\
\leqq & C\left(\int_{0}^{t}\left(\left|\phi_{x}(\tau, 0)\right|^{2}+\left\|\psi_{x}\right\|_{H^{1}}^{2}\right) \mathrm{d} \tau\right)^{\frac{1}{2}}\left(\int_{0}^{t}|\psi(\tau, 0)|^{2} \mathrm{~d} \tau\right)^{\frac{1}{2}} \\
& +C \delta^{2}+C \delta \int_{0}^{t}\left(|\phi(\tau, 0)|^{2}+|\psi(\tau, 0)|^{2}\right) \mathrm{d} \tau \\
\leqq & C\left(\left\|\left(\phi_{0}, \psi_{0}\right)\right\|^{2}+\delta^{\theta}(N(t)+M(t))+\delta^{2}\right) .
\end{aligned}
$$


Furthermore, using again the first energy estimate, Lemma 2.2 and estimate (5.13), we have

$$
\begin{aligned}
& \left|\int_{0}^{t}\left(\psi_{x}, \phi_{t}\right) d \tau\right| \\
& \leq C \int_{0}^{t}\left(\left\|\psi_{x}\right\|^{2}+\varepsilon\left\|\phi_{x}\right\|^{2}\right) \mathrm{d} \tau+C\left(\int_{0}^{t}\left\|\psi_{x}\right\|^{2} \mathrm{~d} \tau\right)^{\frac{1}{2}} \\
& \left.\quad \times \int_{0}^{t}\left(\left\|\tilde{\rho}_{x x}\right\|^{2}+\left\|\tilde{\rho}_{x}\right\|_{4}^{4}+\|\phi\|^{2}\left\|\tilde{u}_{x}\right\|_{\infty}^{2}+\|\psi\|^{2}\left\|\tilde{\rho}_{x}\right\|_{\infty}^{2}\right) \mathrm{d} \tau\right)^{\frac{1}{2}} \\
& \leqq \\
& \quad \int_{0}^{t}\left\|\phi_{x}\right\|^{2} d \tau+C\left(\left\|\left(\phi_{0}, \psi_{0}\right)\right\|^{2}+(\delta+N) M^{2}(t)+\delta^{\theta}(N+M)(t)+\delta^{2}\right) .
\end{aligned}
$$

We now estimate the right-hand side of (5.10). From (3.9) and Lemma 2.2 it follows that

$$
\begin{aligned}
& \left|\int_{0}^{t} \int_{0}^{\infty} \frac{G \phi_{x}}{\rho} \mathrm{d} x \mathrm{~d} \tau\right| \\
& \quad \leqq C\left(\int_{0}^{t}\left\|\phi_{x}\right\|^{2} \mathrm{~d} \tau\right)^{\frac{1}{2}}\left(\int_{0}^{t}\left(\|\phi\|^{2}\left\|\tilde{\rho}_{x}\right\|_{\infty}^{2}+\left\|\psi_{x}\right\|^{2}\left\|\tilde{u}_{x}\right\|_{\infty}^{2}+\left\|\tilde{u}_{x x}\right\|_{\infty}^{2}\right) \mathrm{d} \tau\right)^{\frac{1}{2}} \\
& \quad \leqq C \delta^{\theta} M(t) .
\end{aligned}
$$

Finally, for term of $F_{2}$, recalling (5.5) and (5.6) and the decay estimates of the smoothed rarefaction waves $\tilde{\rho}, \tilde{u}$ as in Lemma 2.2, we then obtain

$$
\begin{aligned}
\left|\int_{0}^{t} \int_{0}^{\infty} \frac{F_{2} \phi_{x}}{\rho} \mathrm{d} x \mathrm{~d} \tau\right| \leqq & C \int_{0}^{t}\left\|\phi_{x}\right\|^{2}\left(\left\|\psi_{x}\right\|_{\infty}+\left\|\tilde{u}_{x}\right\|_{\infty}\right) \mathrm{d} \tau+C\left(\int_{0}^{t}\left\|\phi_{x}\right\|^{2} \mathrm{~d} \tau\right)^{\frac{1}{2}} \\
& \times\left(\int _ { 0 } ^ { t } \left(\left\|\tilde{\rho}_{x x x}\right\|^{2}+\left\|\tilde{\rho}_{x}\right\|_{\infty}^{2}\left\|\tilde{\rho}_{x x}\right\|^{2}+\left\|\tilde{\rho}_{x}\right\|_{6}^{6}+\|\phi\|_{\infty}^{2}\left\|\tilde{u}_{x x}\right\|^{2}\right.\right. \\
& \left.\left.+\|\psi\|_{\infty}^{2}\left\|\tilde{\rho}_{x x}\right\|^{2}+\left\|\phi_{x}\right\|^{2}\left\|\tilde{u}_{x}\right\|_{\infty}^{2}+\left\|\psi_{x}\right\|^{2}\left\|\tilde{\rho}_{x}\right\|^{2}\right) \mathrm{~d} \tau\right)^{\frac{1}{2}} \\
\leqq & C\left((N(t)+\delta) M(t)^{2}+\delta(N(t)+1) M(t)\right) \\
\leqq & C\left((N(t)+\delta) M(t)^{2}+\delta M(t)\right) .
\end{aligned}
$$

Making use of (5.11)-(5.17) and taking $\varepsilon$ suitably small, we then arrive at (5.1). Thus the proof of Lemma 5.1 is complete.

Step 3. Therefore, we can find from the above that to complete the proof of Proposition 4.1 it needs only to show the following Lemma 
Lemma 5.2. There exists a positive constant $\varepsilon_{3} \leqq \varepsilon_{2}$ such that if $N(t)+\delta \leqq \varepsilon_{3}$. Then the following estimate holds for any $t \geqq 0$

$$
\begin{aligned}
\left\|\psi_{x}(t)\right\|^{2}+\int_{0}^{t}\left\|\psi_{x x}(\tau)\right\|^{2} d \tau \leqq & C\left\|\left(\phi_{0}, \psi_{0}\right)\right\|_{H^{1}}^{2}+C\left((N(t)+\delta) M(t)^{2}\right. \\
& \left.+\delta^{\theta}(M(t)+N(t))+\delta^{2}\right)
\end{aligned}
$$

Here $\varepsilon_{3}, C$ are independent of $t, \delta$.

Proof. Multiplying Equation (3.3) by $-\psi_{x x} \rho^{-1}$ and integrating it with respect to $x, t$, we have

$$
\begin{aligned}
& \int_{0}^{t}\left(\psi_{t}+u \psi_{x},-\psi_{x x}\right) \mathrm{d} \tau+\int_{0}^{t} \int_{0}^{\infty} \frac{\mu\left|\psi_{x x}\right|^{2}}{\rho} \mathrm{d} x \mathrm{~d} \tau \\
& -\int_{0}^{t} \int_{0}^{\infty} \frac{p^{\prime}(\rho)}{\rho} \phi_{x} \psi_{x x} \mathrm{~d} x \mathrm{~d} \tau=-\int_{0}^{t} \int_{0}^{\infty} \frac{G \psi_{x x}}{\rho} \mathrm{d} x \mathrm{~d} \tau .
\end{aligned}
$$

It is easy to check that

$$
\left(\psi_{t},-\psi_{x x}\right)=\left.\left(\psi_{t} \psi_{x}\right)\right|_{x=0}+\left(\psi_{x}, \psi_{t x}\right)=\left.\left(\tilde{u}_{t} \psi_{x}\right)\right|_{x=0}+\frac{1}{2} \frac{\mathrm{d}}{\mathrm{d} t}\left\|\psi_{x}\right\|^{2}
$$

Therefore, (5.19) becomes

$$
\begin{gathered}
\frac{1}{2}\left\|\psi_{x}(t)\right\|^{2}+\int_{0}^{t}\left(\left.\tilde{u}_{t} \psi_{x}\right|_{x=0}+\left(u \psi_{x},-\psi_{x x}\right)\right) \mathrm{d} \tau+\int_{0}^{t} \int_{0}^{\infty} \frac{\mu\left|\psi_{x x}\right|^{2}}{\rho} \mathrm{d} x \mathrm{~d} \tau \\
-\int_{0}^{t} \int_{0}^{\infty} \frac{p^{\prime}(\rho)}{\rho} \phi_{x} \psi_{x x} \mathrm{~d} x \mathrm{~d} \tau=\frac{1}{2}\left\|\psi_{0 x}\right\|^{2}-\int_{0}^{t} \int_{0}^{\infty} \frac{G \psi_{x x}}{\rho} \mathrm{d} x \mathrm{~d} \tau
\end{gathered}
$$

We now handle (5.21) term by term. We first use Lemma 2.2 and Hölder's inequality to estimate the boundary term as follows:

$$
\begin{aligned}
\left|\int_{0}^{t}\left(\tilde{u}_{t} \psi_{x}\right)(\tau, 0) \mathrm{d} \tau\right| & \leqq C\left(\int_{0}^{t}\left|\tilde{u}_{t}(\tau, 0)\right|^{2} \mathrm{~d} \tau\right)^{\frac{1}{2}}\left(\int_{0}^{t}\left|\psi_{x}(\tau, 0)\right|^{2} \mathrm{~d} \tau\right)^{\frac{1}{2}} \\
& \leqq C \delta\left(\int_{0}^{t}\left\|\psi_{x}\right\|_{H^{1}}^{2} \mathrm{~d} \tau\right)^{\frac{1}{2}} \\
& \leqq C \delta M(t) .
\end{aligned}
$$

Moreover we can show that

$$
\begin{aligned}
\left|\int_{0}^{t} \int_{0}^{\infty} u \psi_{x}\left(-\psi_{x x}\right) \mathrm{d} x \mathrm{~d} \tau\right| & \leqq \varepsilon \int_{0}^{t}\left\|\psi_{x x}\right\|^{2} \mathrm{~d} \tau+C_{\varepsilon} \int_{0}^{t}\left\|\psi_{x}\right\|^{2} \mathrm{~d} \tau \\
\int_{0}^{t} \int_{0}^{\infty} \frac{\mu\left|\psi_{x x}\right|^{2}}{\rho} \mathrm{d} x \mathrm{~d} \tau & \geqq C\left\|\psi_{x x}\right\|^{2}
\end{aligned}
$$


and

$$
\begin{aligned}
\left|\int_{0}^{t} \int_{0}^{\infty} \frac{p^{\prime}(\rho)}{\rho} \phi_{x} \psi_{x x} \mathrm{~d} x \mathrm{~d} \tau\right| & \leqq C \int_{0}^{t} \int_{0}^{\infty}\left|\phi_{x}\right|\left|\psi_{x x}\right| \mathrm{d} x \mathrm{~d} \tau \\
& \leqq \varepsilon \int_{0}^{t}\left\|\psi_{x x}\right\|^{2} \mathrm{~d} \tau+C_{\varepsilon} \int_{0}^{t}\left\|\phi_{x}\right\|^{2} \mathrm{~d} \tau
\end{aligned}
$$
obtain

Finally we estimate the right-hand side in a similar way as we handle (5.16) to

$$
\begin{aligned}
\left|\int_{0}^{t} \int_{0}^{\infty} \frac{G \psi_{x x}}{\rho} \mathrm{d} x \mathrm{~d} \tau\right| \leqq & C\left(\int_{0}^{t}\left\|\psi_{x x}\right\|^{2} \mathrm{~d} \tau\right)^{\frac{1}{2}} \\
& \times\left(\int_{0}^{t}\left(\|\phi\|^{2}\left\|\tilde{\rho}_{x}\right\|_{\infty}^{2}+\left\|\psi_{x}\right\|^{2}\left\|\tilde{u}_{x}\right\|_{\infty}^{2}+\left\|\tilde{u}_{x x}\right\|_{\infty}^{2}\right) \mathrm{d} \tau\right)^{\frac{1}{2}} \\
\leqq & C \delta^{\theta} M(t) .
\end{aligned}
$$

Therefore, combination of Equations (5.21)-(5.26) and taking $\varepsilon$ suitably small, we then have

$$
\begin{aligned}
\left\|\psi_{x}\right\|^{2}+\int_{0}^{t}\left\|\psi_{x x}\right\|^{2} \mathrm{~d} \tau \leqq & C\left\|\psi_{0}\right\|_{H^{1}}^{2}+C \int_{0}^{t}\left(\left\|\phi_{x}\right\|^{2}+\left\|\psi_{x}\right\|^{2}\right) \mathrm{d} \tau \\
& +C\left(\delta M(t)+\delta^{\theta} M(t)\right) .
\end{aligned}
$$

Thus (5.18) follows from (5.1) and the first energy estimates. And the proof of this lemma is complete.

Complete of the proof of Proposition 3.2: From the estimates (4.5), (5.1) and (5.18), it follows that there exists a constant $\varepsilon_{4}$ (which satisfies $\varepsilon_{4} \leqq \varepsilon_{3}$, and we can take $\left.\varepsilon_{0}=\varepsilon_{4}\right)$, such that if $N(t)+\delta \leqq \varepsilon_{4}$, then the following estimate holds

$$
\begin{aligned}
N^{2}(t)+M^{2}(t)= & \|(\phi, \psi)\|_{H^{1}}^{2}+\int_{0}^{t}\left(\left\|\phi_{x}\right\|^{2}+\left\|\psi_{x}\right\|_{H^{1}}^{2}+|\phi(\tau, 0)|^{2}+\left|\phi_{x}(\tau, 0)\right|^{2}\right) \mathrm{d} \tau \\
\leqq & C\left(\left\|\left(\phi_{0}, \psi_{0}\right)\right\|_{H^{1}}^{2}+\delta^{2}\right) \\
& +C_{1}\left((N(t)+\delta) M(t)^{2}+\delta^{\theta}(N(t)+M(t))\right) .
\end{aligned}
$$

Therefore, if we take $\varepsilon_{4}<1$ such that $C_{1} \varepsilon_{4} \leqq \frac{1}{4}$, using the Young inequality one has

$$
N^{2}(t)+M^{2}(t) \leqq C\left(\left\|\left(\phi_{0}, \psi_{0}\right)\right\|_{H^{1}}^{2}+\delta^{2 \theta}\right)+\frac{1}{2}\left(N^{2}(t)+M^{2}(t)\right) .
$$

That is

$$
\begin{aligned}
& \|(\phi, \psi)(t)\|_{H^{1}}^{2}+\int_{0}^{t}\left(\left\|\phi_{x}(\tau)\right\|^{2}+\left\|\psi_{x}(\tau)\right\|_{H^{1}}^{2}+|\phi(\tau, 0)|^{2}+\left|\phi_{x}(\tau, 0)\right|^{2}\right) \mathrm{d} \tau \\
& \quad \leqq C\left(\left\|\left(\phi_{0}, \psi_{0}\right)\right\|_{H^{1}}^{2}+\delta^{2 \theta}\right) .
\end{aligned}
$$

Which implies the results of Proposition 3.2. Thus the proof is complete. 


\section{The proof of Theorem 1.1}

This section is devoted to the proof of our main theorem. In order to prove Theorem 1.1, we employ the standard continuation argument based on a local existence theorem and the a priori estimates. Similar to [10], we can prove easily the local existence theorem. The a priori estimates have been established in Proposition 3.2. Therefore, we need only to prove the large time behavior of the solution as $t \rightarrow \infty$. For a rigorous argument we refer also to [10].

Complete of the proof of Theorem 1.1:

Step 1. We first make use of the energy estimates to prove that

$$
\sup _{x \in \mathbb{R}^{+}}|(\rho-\tilde{\rho}, u-\tilde{u})(t, x)| \rightarrow 0,
$$

namely

$$
\sup _{x \in \mathbb{R}^{+}}|(\phi, \psi)(t, x)| \rightarrow 0
$$

as $t \rightarrow \infty$. To this end, we only need to show that

$$
\left\|\phi_{x}(t)\right\|, \quad\left\|\psi_{x}(t)\right\| \rightarrow 0 .
$$

If this holds, recalling the estimates $\|\phi\|_{H^{1}}$ and $\|\psi\|_{H^{1}} \leqq C$, then by interpolation, we arrive at

$$
\begin{aligned}
\|\phi(t)\|_{\infty} & \leqq C\|\phi(t)\|^{\frac{1}{2}}\left\|\phi_{x}(t)\right\|^{\frac{1}{2}} \\
& \rightarrow 0, \quad \text { as } t \rightarrow \infty .
\end{aligned}
$$

Similarly, we can infer from $\left\|\psi_{x}(t)\right\| \rightarrow 0$ that $\|\psi(t)\|_{\infty} \rightarrow 0$ as $t \rightarrow \infty$. So, it remains to show (6.3). We define

$$
P(t)=\int \frac{\left(\phi_{x}\right)^{2}}{\rho^{2}}(t, x) \mathrm{d} x, \quad U(t)=\int\left(\psi_{x}\right)^{2}(t, x) \mathrm{d} x .
$$

It is easy to obtain from the energy estimates that

$$
\int_{0}^{\infty} P(t) \mathrm{d} t \leqq C
$$

Next to prove $\int_{0}^{\infty}\left|\frac{\mathrm{d}}{\mathrm{d} t} P(t)\right| \mathrm{d} t \leqq C$, we invoke Equation (5.10), and conclude that the function $P(t):=\left\|\frac{\phi_{x}}{\rho}(t)\right\|^{2}$ is differentiable with respect to $t$ for almost everywhere $t \in(0, \infty)$. Thus by combining with the estimates (5.7) and (5.29) we can obtain

$$
\int_{0}^{\infty}\left|\frac{\mathrm{d}}{\mathrm{d} t}\left\|\frac{\phi_{x}}{\rho}(t)\right\|^{2}\right| \mathrm{d} t \leqq C .
$$

That is

$$
\int_{0}^{\infty}\left|\frac{\mathrm{d}}{\mathrm{d} t} P(t)\right| \mathrm{d} t \leqq C
$$


Recalling the fact $\int_{0}^{\infty} P(t) \mathrm{d} t \leqq C$, one thus has $P(t) \rightarrow 0$; therefore $\left\|\phi_{x}(t)\right\| \rightarrow 0$ as $t \rightarrow \infty$.

We can use Equation (5.21) and the energy estimates to show, in a similar way, that $\left\|\psi_{x}(t)\right\| \rightarrow 0$.

Step 2. On the other hand, by the construction of a smooth approximation of the rarefaction waves, from Lemma 2.2 and the results in [12], we infer that

$$
\sup _{x \in \mathbb{R}^{+}}\left|(\tilde{\rho}, \tilde{u})(t, x)-\left(\rho^{R}, u^{R}\right)\left(\frac{x}{t}\right)\right| \rightarrow 0,
$$

as $t \rightarrow \infty$.

It then follows from (6.1) and (6.5) that

$$
\sup _{x \in \mathbb{R}^{+}}\left|(\rho, u)(t, x)-\left(\rho^{R}, u^{R}\right)\left(\frac{x}{t}\right)\right| \rightarrow 0
$$

as $t \rightarrow \infty$. Thus the proof of Theorem 1.1 is complete.

\section{References}

1. FreistüHler, H., Serre, D.: $L^{1}$-stability of shock waves in scalar viscous conservation laws. Commun. Pure Appl. Math. 51, 291-301 (1998)

2. Friedman, A.: Partial Differential Equations of Parabolic Type. Prentice Hall, Inc. Englewood Cliffs, 1964

3. Goodman, J.: Nonlinear asymptotic stability of viscous shock profiles for conservation laws. Archive Rat. Mech. Anal. 95, 325-344 (1986)

4. Huang, F., Matsumura, A., Shi, X.: Asymptotics toward the viscous shock wave to an inflow problem in the half space for the compressible viscous gas. Commun. Math. Phys. 239, 261-285 (2003)

5. IL'In, A., OleiniK, O.: Asymptotic behavior of the solutions of Cauchy problems for certain quasilinear equations for large time (Russian). Mat. Sbornik 51, 191-216 (1960)

6. Jones, C.K.R.T., GARDER, R., KAPITUla, T.: Stability of traveling waves for non-convex scalar viscous conservation laws. Commun. Pure Appl. Math. 46, 505-526 (1993)

7. Kawashima, S., Matsumura, A.: Asymptotic stability of traveling waves solutions of systems for one-dimensional gas motion. Commun. Math. Phys. 101, 97-127 (1985)

8. Kawashima, S., Matsumura, A.: Stability of shock profiles in viscoelasticity with non-convex constitutive relations. Commun. Pure Appl. Math. 47, 1547-1569 (1994)

9. Kawashima, S., NikKuni, Y.: Stability of rarefaction waves for the discrete Boltzman equations. Adv. Math. Sci. Appl. 12(1), 327-353 (2002)

10. Kawashima, S., Nishibata, S., Zhu, P.: Asymptotic stability of the stationary solution to the compressible Navier-Stokes equations in the half space. Commun. Math. Phys. 240(3), 483-500 (2003)

11. Kawashima, S., Nishida, T.: Global solutions to the initial value problem for the equations of one-dimensional motion of viscous polytropic gases. J. Math. Kyoto Univ. (JMKYAZ) 21-4, 825-837 (1981)

12. Kawashima, S., Tanaka, Y.: Stability of rarefaction waves for a model system of a radiating gas. Kyushu J. Math. 58, 211-250 (2004)

13. Kawashima, S., Zhu, P.: Asymptotic stability of nonlinear wave for the compressible Navier-Stokes equations in the half space. J. Differ. Equ. 244, 3151-3179 (2008)

14. Kawashima, S., Nakamura, T., Nishibata, S., Zhu, P.: Existence and asymptotic stability of stationary solution to the full compressible Navier-Stokes equations in the half space. (in preparation)

15. LiU, T.: Nonlinear stability of shock waves for viscous conservation laws. Mem. AMS $56(1985)$ 
16. Liu, T.: Nonlinear waves for viscous conservation laws. Nonlinear evolutionary partial differential equations (Beijing, 1993). AMS/IP Stud. Adv. Math., vol. 3, pp. 65-74. Am. Math. Soc., Providence, 1997

17. Liu, T., Matsumura, A., Nishinara, K.: Behavior of solutions for the Burgers equations with boundary corresponding to rarefaction waves. SIAM J. Math. Anal. 29, 293-308 (1998)

18. Liu, T., Nishinara, K.: Asymptotic behavior for scalar viscous conservation laws with boundary effect. J. Differ. Equ. 133, 296-320 (1997)

19. Liu, T., YU, S.: Propagation of stationary viscous Burger shock under the effect of boundary. Archive Rat. Mech. Anal. 139, 57-82 (1997)

20. LiU, T., XIN, Z.: Asymptotic stability of the rarefaction waves of compressible NavierStokes equations. Commun. Math. Phys. 118, 325-335 (1986)

21. Matsumura, A.: Inflow and outflow problems in the half space for a one-dimensional isentropic model system for compressible viscous gas. In: Proceedings of IMS Conference on Differential Equations from Mechanics, Hongkong, 1999

22. Matsumura, A., Nishihara, K.: On stability of traveling waves solutions of a onedimensional model system for compressible viscous gas. Jpn. J. Appl. Math. 3, 17-25 (1985)

23. Matsumura, A., Nishihara, K.: Asymptotics towards the rarefaction waves of the solutions of a one-dimensional model system for compressible viscous gas. Jpn. J. Appl. Math. 3, 1-13 (1986)

24. Matsumura, A., Nishinara, K.: Global stability of the rarefaction waves of a onedimensional model system for compressible viscous gas. Commun. Math. Phys. 144, 325-335 (1992)

25. Matsumura, A., Nishinara, K.: Global asymptotics towards the rarefaction waves for the solutions of viscous $p$-system with boundary effect. Q. Appl. Math. 58, 69-83 (2000)

26. Matsumura, A., Nishihara, K.: Large-time behaviors of solutions to an inflow problem in the half space for a one-dimensional isentropic model system for compressible viscous gas (in preprint)

27. Matsumura, A., Nishinara, K.: Asymptotic stability of traveling waves for scalar viscous conservation laws with non-convex nonlinearity. Commun. Math. Phys. 165, 83-96 (1994)

28. Matsumura, A., Mei, M.: Convergence to traveling front of solutions of the $p$-system with viscosity in the presence of a boundary. Archive Rat. Mech. Anal. 146, 1-22 (1999)

29. MeI, M.: Stability of shock profiles for non-convex scalar viscous conservation laws. Math. Models Methods Appl. Sci. 5, 27-35 (1995)

30. ZHU, P.: Existence and asymptotic stability of stationary solution to the full compressible Navier-Stokes equations in the half space. Mathematical analysis in fluid and gas dynamics, RIMS Kokyuroku, vol. 1247, pp. 187-207, 2002

31. ZHU, P.: Nonlinear waves for the compressible Navier-Stokes equations in the half space. Ph.D. Thesis, Kyushu University, Fukuoka, 2001

Graduate School of Mathematics, Kyushu University,

Fukuoka 812-8581, Japan

and

Technical University of Darmstadt, Darmstadt, Germany.

e-mail: zhu@mathematik.tu-darmstadt.de 
and

Basque Center for Applied Mathematics,

Gran Via 35-2,

48009 Bilbao, Spain.

e-mail: zhu@bcamath.org

(Received December 7, 2006 / Accepted March 12, 2008)

Published online November 20, 2008 - (C) Springer-Verlag (2008) 